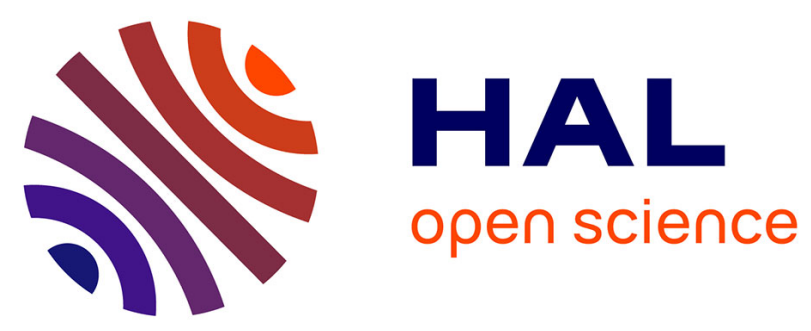

\title{
Uniformly Projected RCQD QAM: A Low-Complexity Signal Space Diversity Solution over Fading Channels With or Without Erasures
}

Tarak Arbi, Benoit Geller, Jianxiao Yang, Charbel Abdel Nour, Olivier Rioul

\section{- To cite this version:}

Tarak Arbi, Benoit Geller, Jianxiao Yang, Charbel Abdel Nour, Olivier Rioul. Uniformly Projected RCQD QAM: A Low-Complexity Signal Space Diversity Solution over Fading Channels With or Without Erasures. IEEE Transactions on Broadcasting, 2018, 10.1109/TBC.2018.2811618 . hal01756306

\section{HAL Id: hal-01756306 \\ https://hal.science/hal-01756306}

Submitted on 1 Apr 2018

HAL is a multi-disciplinary open access archive for the deposit and dissemination of scientific research documents, whether they are published or not. The documents may come from teaching and research institutions in France or abroad, or from public or private research centers.
L'archive ouverte pluridisciplinaire HAL, est destinée au dépôt et à la diffusion de documents scientifiques de niveau recherche, publiés ou non, émanant des établissements d'enseignement et de recherche français ou étrangers, des laboratoires publics ou privés. 


\title{
Uniformly Projected RCQD QAM: A Low-Complexity Signal Space Diversity Solution over Fading Channels With or Without Erasures
}

\author{
Tarak Arbi, Benoit Geller, Jianxiao Yang, Charbel Abdel Nour and Olivier Rioul
}

\begin{abstract}
Rotated and cyclic Q-delayed (RCQD) $M$-QAM (Quadrature Amplitude Modulation) provides signal space diversity and thus improves system performance over fading channels. However, previously published RCQD solutions were designed without fully considering the high demodulation complexity which prohibits wider applications. In this paper, a complete solution is proposed to reduce complexity for both the modulator and the demodulator. This solution uses a series of rotation angles $\alpha=\arctan (1 / \sqrt{M})$ which bring many interesting properties to the RCQD signals. A simplified sphere demapping algorithm is derived for fading channels with and without erasure events. In contrast to the sphere-decoder used for MIMO detection, the radius of the proposed sphere-demapper involves an exact amount of constellation points, thereby ensuring to perform the soft demapping operation successfully. Moreover, when either the in-phase (I) or the quadrature component (Q) is erased, the proposed demapping algorithm performs as well as the fullcomplexity Max-Log algorithm, with a reduced complexity. Compared to the solution currently used in DVB-T2, the proposed method reduces tremendously the computational complexity while still achieving similar performance over fading channels and even better performance over fading erasure channels.
\end{abstract}

Index Terms-Rotated and Cyclic Q-Delayed (RCQD), Quadrature Amplitude Modulation (QAM), DVB-T2, Signal Space Diversity (SSD), Fading Channel, Demodulation Complexity, Sphere-Demapper, Fading Erasure Channel, Broadcasting System.

\section{INTRODUCTION}

$\mathbf{R}$ OTATED and cyclic Q-delayed (RCQD) modulations or signal space diversity (SSD) techniques [1]-[4] outperform conventional QAM signals over fading channels [5]-[8]. Due to its excellent performance, the DVB-T2 standard [9] was the first industrial standard to deploy the RCQD modulation $[10,11]$. The key idea behind this technique can be outlined as follows; the rotation correlates the in-phase (I) and quadrature (Q) components of a conventional $M$-QAM so that each component of the rotated signal carries complete information of the $M$-QAM signal; these two components are then distributed through independent fading subchannels by the cyclic Q-delay. Thanks to an increased signal space diversity, the performance of RCQD outperforms the conventional $M$-QAM over various

Tarak Arbi, Benoit Geller and Jianxiao Yang are with Department U2IS, of ENSTA-ParisTech, 828 Boulevard des Marechaux, 91120, Palaiseau, France (e-mail: tarak.arbi,benoit.geller,jianxiao.yang@ensta-paristech.fr);

Charbel Abdel Nour is with Electronics department, IMT Atlantique, CNRS Lab-STICC UMR 6285 Technopôle Brest-Iroise, CS 83818, 29238 Brest Cedex 3, France (e-mail: charbel.abdelnour@imt-atlantique.fr);

Olivier Rioul is with LTCI, Telecom ParisTech, 75013, Paris, France (email: olivier.rioul@telecom-paristech.fr). fading environments [2]. But the corresponding complexity of the optimal maximum likelihood (ML) demapper increases to an order $\mathrm{O}(M)$ instead of the complexity order $\mathrm{O}(\sqrt{M})$ for conventional $M$-QAM signals.

To reduce the complexity, suboptimum Max-Log-based demappers are generally used. Decorrelation-based methods such as zero-forcing ( $\mathrm{ZF}$ ) or minimum mean square error (MMSE) demappers [12] are frequently encountered. In [12], the channel attenuations and rotation are treated as an equivalent $2 \times 2$ multiple input multiple output (MIMO) channel response matrix, and MMSE equalization is used to decorrelate the I and Q components. The equalized symbols can then be demapped as conventional $M$-QAM symbols with an $\mathrm{O}(\sqrt{M})$ complexity, but the noise level is increased and performance becomes poor when the $2 \times 2$ channel response matrix is singular. Instead, two-dimensional (I and Q) demappers (2D-DEM) [13]-[16] approach much closely the optimum performance. In [13], a QAM constellation space is decomposed into four squared sub-regions and the demapping is performed within one of the four sub-regions according to the signs of the real and imaginary parts of the equalized symbol. Although this method reduces the computation complexity for a $M$ QAM modulation and has better performance than [12], the demapping complexity is still high with an $\mathrm{O}(M)$ complexity. In [14], the authors divide the constellation space into $2 \sqrt{M}$ sub-regions for the I and Q components respectively, and select two subregions for each bit of the QAM symbol according to the equalized symbol. This method is simpler than [13], however, due to the low dependence among the bits of one QAM symbol, the total number of points to perform demapping for one symbol, is approximatively equal to $\sqrt{M}\left(\left(\log _{2} M\right) / 2\right)$, which is actually comparable to the complexity of [13]. In [15], a sub-region based method is proposed, which divides the constellation plane into 16 independent sub-quadrants. For each sub-quadrant, [15] constructs a histogram of constellation points participating to the Max-Log LLR computation. Therefore, there are 16 histograms used for demapping with their associated complexities and the number of constellation points participating to the demapping depends on the signal to noise ratio (SNR). In [27] a reduced complexity soft demapper is proposed, which selects a set of $2 \sqrt{M}$ constellation points. The LLR values are then computed within this set. Their proposed $\sqrt{M}$-Best demapper achieves the same performance as the Max-Log algorithm with a reduced complexity.

In contrast to previous works $[2,3,4,10,11,17,18,19$, $20,21]$ which focused only on the transmitter side, [22] 
put forth a series of rotation angles to greatly simplify the demapper's design and led recently to a proposal of hardware implementation [23]. This series of angles was independently proposed by [28, 29] without considering the demapping and complexity issues. In addition, neither [22], nor [28, 29] give any proof of the properties introduced by this series of angles. In this paper, we first elaborate on [22] to systematically prove all of the algebraic properties introduced by the considered series of angles and show how they allow the simplification of the demapper. In particular, the fact that the studied rotated constellations keep the uniformity of the non-rotated constellations, enables simple mutual conversions between each projected point over the I or Q axis and its corresponding 2D QAM constellation point. Taking advantage of this structural property, a low-complexity sphere-demapper is then proposed. Different from the sphere decoders [24] used for MIMO systems, the radius of the proposed spheredemapper indicates the number of constellation points to be considered and guarantees to perform successful soft demapping. We also go beyond [22] by proving that for the fading case with one erased component, the nearest constellation point to the observation symbol (also known as the ML point) is always found. We demonstrate that in this case the demapper has the same performance as the Max-Log algorithm. In contrast to [27], the proposed demapper takes advantage of several specific properties of the $2 \sqrt{M}$ points involved in the LLR computation; this simplifies the selection of these points for fading channels without erasure; this also reduces the complexity significantly for fading channels with erasures, since we prove that $\sqrt{M}$ candidate points are sufficient to obtain the same performance as the Max-Log algorithm for the Gray-mapped UP-RCQD. It can be noticed that the proposed demapper is based on the concept of UP-RCQD and cannot as such be applied to systems such as ASTC 3.0 which do not use uniform constellations. The complete solution is then applied to the DVB-T2 system and compared with the original rotation angles used in the standard for various constellations. In particular, the studied angle is the same angle as the one used in DVB-T2 in the sole case of the 256-QAM constellation and this allows illustrating the considerable complexity reduction of our demapper. Despite this simplification, it achieves the same performance as the original solution over fading channels for various constellations, and even outperforms the original solution over fading channels in the presence of erasure events. In addition, we also provide several interpretations for the high performance of this proposal over fading channels with and without erasure events. Note that the displayed results are obtained with LDPC codes but similar results can similarly be obtained with other near-capacity channel codes [30], [31].

The remainder of this article is organized as follows. Section II sketches a brief review of the RCQD modulation and demodulation. In Section III, the proposed low-complexity RCQD SSD solution is detailed. A comparison in terms of computational complexity is made and simulation results are compared to previous techniques for different constellations

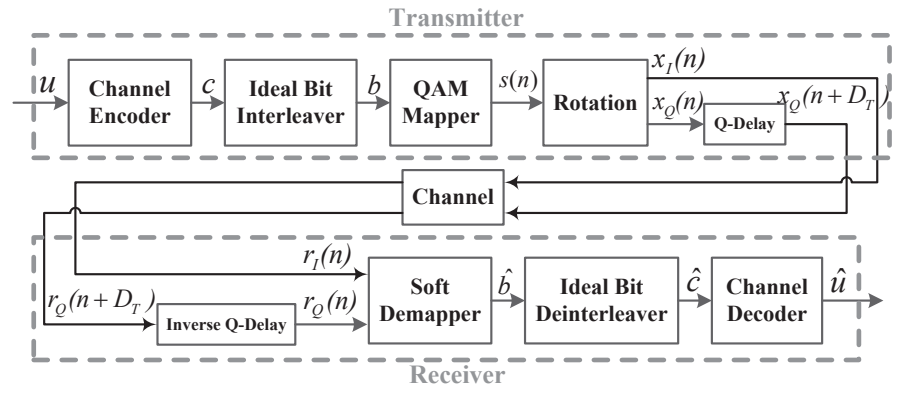

Figure 1. BICM system model with RCQD constellation

over different fading environments in Section IV. Finally, some conclusions are drawn in Section V.

\section{System Model}

A BICM system model with RCQD constellation is displayed in Fig. 1. We consider square $M$-QAM signals for which $N=\sqrt{M}$ is an integer whose value is a power of 2 .

\section{A. The M-QAM RCQD Signal Transmission}

A message frame $u$ passes through a channel encoder and generates a codeword $c$. The codeword is then interleaved to guarantee that consecutive bits are transmitted through independent fading subchannels.

After interleaving, every consecutive $\log _{2} M$ bits are Graymapped to a conventional complex-valued $M$-QAM symbol:

$$
s(n)=s_{I}(n)+j s_{Q}(n),
$$

where $s_{I}(n)$ and $s_{Q}(n)$ are the Gray-mapped in-phase (I) and quadrature $(\mathrm{Q})$ components of $s(n)$ at time $n$; the two components can be expressed as:

$$
\begin{gathered}
s_{I}(n)=2 p_{I}-N+1, \\
s_{Q}(n)=2 p_{Q}-N+1,
\end{gathered}
$$

where $p_{I}$ and $p_{Q}$ take integer values in the set $A_{c}=$ $\{0,1, \ldots, N-1\}$. Note that in compliance with the Gray mapping defined in DVB-T2 [9], $s_{I}(n)$ (or $p_{I}$ ) is mapped from the even-indexed bits whereas $s_{Q}(n)$ (or $p_{Q}$ ) is mapped from the odd-indexed bits.

The first step of the RCQD modulation is to correlate $s_{I}(n)$ and $s_{Q}(n)$ so that the in-phase component $x_{I}(n)$ and quadrature component $x_{Q}(n)$ of the rotated symbol $x(n)$ contain the complete information of the original unrotated symbol $s(n)$, through the multiplication with a properly designed rotation factor $\exp (j \alpha)$ :

$$
\begin{aligned}
x(n) & =x_{I}(n)+j x_{Q}(n)=s(n) \exp (j \alpha) \\
& =\left(s_{I}(n) \cos \alpha-s_{Q}(n) \sin \alpha\right)+j\left(s_{I}(n) \sin \alpha+s_{Q}(n) \cos \alpha\right) .
\end{aligned}
$$

The second step of the RCQD modulation is to cyclically delay the $\mathrm{Q}$ component $x_{Q}(n)$ so that the components $x_{I}(n)$ and $x_{Q}(n)$ of the rotated symbol $x(n)$ pass through independently attenuated subchannels as follows:

$$
\begin{array}{r}
z(n)=\beta\left(x_{I}(n)+j x_{Q}\left(n+D_{T}\right)\right), \\
z\left(n-D_{T}\right)=\beta\left(x_{I}\left(n-D_{T}\right)+j x_{Q}(n)\right),
\end{array}
$$


where $\beta$ is the normalization factor of the QAM constellation (e.g., $\beta=1 / \sqrt{170}$ for a 256-QAM signal) and $D_{T}$ is an integer delay factor. Terrestrial broadcasting systems generally use the orthogonal frequency-division multiplexing (OFDM) modulation. This enables the use of a simple channel equalizer, where the received attenuated symbol $y(n)$ over one OFDM subcarrier can be written as:

$$
y(n)=H(n) z(n)+w(n)
$$

where $H(n)$ is the known complex-valued fading attenuation factor whose module $|H(n)|$ is Rayleigh-distributed with unit variance, and $w(n)$ is a zero-mean complex-valued additive white Gaussian noise (AWGN) with known noise variance $\sigma^{2}$. For a fading channel model with erasure events [25], the attenuation $H(n)$ is equal to zero if an erasure event happens.

To extract the $\mathrm{I}$ and $\mathrm{Q}$ components $x_{I}(n)$ and $x_{Q}(n)$ of $x(n)$ from different received symbols $y(n)$ and $y\left(n-D_{T}\right)$ respectively, some reverse operations need to be performed:

$$
\begin{aligned}
& r_{I}(n)=\operatorname{Re}\left\{y(n) e^{-j \arg \{H(n)\}}\right\} \\
& =\beta \operatorname{Re}\left\{H(n) e^{-j \arg \{H(n)\}}\left(x_{I}(n)+j x_{Q}\left(n+D_{T}\right)\right)\right\}+v_{I}(n) \\
& =\beta h_{I}(n) x_{I}(n)+v_{I}(n),
\end{aligned}
$$

$$
\begin{aligned}
& r_{Q}(n)=\operatorname{Im}\left\{y\left(n-D_{T}\right) e^{-j \arg \left\{H\left(n-D_{T}\right)\right\}}\right\} \\
& =\beta \operatorname{Im}\left\{H\left(n-D_{T}\right) e^{-j \arg \left\{H\left(n-D_{T}\right)\right\}}\left(x_{I}\left(n-D_{T}\right)+j x_{Q}(n)\right)\right\} \\
& \quad+v_{Q}(n) \\
& =\beta h_{Q}(n) x_{Q}(n)+v_{Q}(n),
\end{aligned}
$$

where $r_{I}(n)$ and $r_{Q}(n)$ are defined as the received observation components of the transmitted components $x_{I}(n)$ and $x_{Q}(n)$, and where $\arg \{a\}$ denotes the argument of the complex number $a$; in (8) and (9) the channel attenuation terms $h_{I}(n)$ and $h_{Q}(n)$, and the noise terms $v_{I}(n)$ and $v_{Q}(n)$ are given by:

$$
\begin{aligned}
h_{I}(n) & =H(n) e^{-j \arg \{H(n)\}}, \\
h_{Q}(n) & =H\left(n-D_{T}\right) e^{-j \arg \left\{H\left(n-D_{T}\right)\right\}}, \\
v_{I}(n) & =\operatorname{Re}\left\{w(n) e^{-j \arg \{H(n)\}}\right\}, \\
v_{Q}(n) & =\operatorname{Im}\left\{w\left(n-D_{T}\right) e^{-j \arg \left\{H\left(n-D_{T}\right)\right\}}\right\} .
\end{aligned}
$$

In order to simplify the notations, in the sequel, the index $(n)$ is dropped.

\section{B. The QAM RCQD Signal Demapping at the Receiver Side}

For each symbol $x$, the soft demapper computes the log-likelihood ratio (LLR) for the mapped bits $l^{i}(x)(i=$

$$
\begin{aligned}
& \left.0,1, \ldots, \log _{2} M-1\right) \text { as: } \\
& \begin{aligned}
\Lambda\left(l^{i}(x)\right) & =\log \left(\sum_{x \in \boldsymbol{\chi}_{0}^{i}} p(r \mid x, h)\right)-\log \left(\sum_{x \in \boldsymbol{\chi}_{1}^{i}} p(r \mid x, h)\right) \\
& =\log \left(\sum_{x \in \boldsymbol{\chi}_{0}^{i}} \exp \left\{-\left(\frac{\left|r_{I}-h_{I} x_{I} \beta\right|^{2}}{\sigma^{2}}+\frac{\left|r_{Q}-h_{Q} x_{Q} \beta\right|^{2}}{\sigma^{2}}\right)\right\}\right) \\
& -\log \left(\sum_{x \in \boldsymbol{\chi}_{1}^{i}} \exp \left\{-\left(\frac{\left|r_{I}-h_{I} x_{I} \beta\right|^{2}}{\sigma^{2}}+\frac{\left|r_{Q}-h_{Q} x_{Q} \beta\right|^{2}}{\sigma^{2}}\right)\right\}\right),
\end{aligned}
\end{aligned}
$$

where $l^{i}(x)$ represents the $\mathrm{i}$-th bit of symbol $x, h$ is the two component vector $\left(h_{I}, h_{Q}\right)$, and $\chi_{b}^{i}$ denotes the set of the constellation points whose i-th bit is $b=0$ or $b=1$. Note that the computation of $\Lambda\left(l^{i}(x)\right)$ requires the computation of M 2D Euclidean distances.

In practice, a sub-optimum max-log demapper [25] is widely used to approximately calculate (14) and simplifies the LLR computation:

$$
\begin{aligned}
\Lambda\left(l^{i}(x)\right) & =\frac{1}{\sigma^{2}} \min _{x \in \boldsymbol{\chi}_{1}^{i}}\left\{\left|r_{I}-h_{I} x_{I} \beta\right|^{2}+\left|r_{Q}-h_{Q} x_{Q} \beta\right|^{2}\right\} \\
& -\frac{1}{\sigma^{2}} \min _{x \in \boldsymbol{\chi}_{0}^{i}}\left\{\left|r_{I}-h_{I} x_{I} \beta\right|^{2}+\left|r_{Q}-h_{Q} x_{Q} \beta\right|^{2}\right\} .
\end{aligned}
$$

Note, however, that all constellation points must be considered to obtain $\Lambda\left(l^{i}(x)\right)$ in (15).

If an erasure event occurs over one of the components, (14) becomes:

$$
\begin{aligned}
\Lambda\left(l^{i}(x)\right) & =\log \left(\sum_{x \in \chi_{0}^{i}} \exp \left(-\frac{2\left|r_{m}-h_{m} x_{m} \beta\right|^{2}}{\sigma^{2}}\right)\right) \\
& -\log \left(\sum_{x \in \chi_{1}^{i}} \exp \left(-\frac{2\left|r_{m}-h_{m} x_{m} \beta\right|^{2}}{\sigma^{2}}\right)\right),
\end{aligned}
$$

where the index $m$ can either be $\mathrm{I}$ or $\mathrm{Q}$ according to the remaining non-erased component.

Similarly to (15), the Max-Log based LLR computation with one erased component can then be written as:

$$
\begin{aligned}
\Lambda\left(l^{i}(x)\right) & =\frac{2}{\sigma^{2}} \min _{x \in \chi_{1}^{i}}\left\{\left|r_{m}-h_{m} x_{m} \beta\right|^{2}\right\} \\
& -\frac{2}{\sigma^{2}} \min _{x \in \boldsymbol{\chi}_{0}^{i}}\left\{\left|r_{m}-h_{m} x_{m} \beta\right|^{2}\right\} .
\end{aligned}
$$

\section{The Uniformly Projected RCQD Modulation AND DEMAPPING SOLUTION}

The studied RCQD solution is based on the choice of a series of rotation angles $\alpha=\arctan (1 / N)$. These angles lead the points of a rotated M-QAM constellation to be uniquely and uniformly projected over the I and Q axes. Therefore, the rotated constellation points can be translated to the integer domain and this enables many important simplifications. 
This section is organized as follows: Subsection A presents the properties of the considered angles. The demapping solution is detailed for fading channels with and without erasure in Subsection B and is summarized in Subsection C. The complexity analysis is finally discussed in Subsection D.

\section{A. Properties of the studied Angles}

With the chosen rotation angle $\alpha=\arctan (1 / N)$, the components $x_{I}$ and $x_{Q}$ of the rotated $M$-QAM signal (4) can be rewritten as:

$$
\begin{aligned}
x_{I} & =\left(N s_{I}-s_{Q}\right) \sin \alpha, \\
x_{Q} & =\left(N s_{Q}+s_{I}\right) \sin \alpha .
\end{aligned}
$$

Property 1. With the rotation angle $\alpha=\arctan (1 / N)$, the I and $Q$ components of the rotated symbols are uniformly projected along the I and $Q$ axes with a constant minimum interval $d_{1 \mathrm{D}, \min }=2 \sin \alpha$ between neighbor projected components.

Proof: From (2)-(3) and (18)-(19) the I and Q components can be written as:

$$
\begin{aligned}
x_{I} & =\left(2\left(N p_{I}+\left(-p_{Q}+N-1\right)\right)-M+1\right) \sin \alpha, \\
x_{Q} & =\left(2\left(N p_{Q}+p_{I}\right)-M+1\right) \sin \alpha .
\end{aligned}
$$

Consider any rotated symbol $x_{1}=\left(x_{I, 1}, x_{Q, 1}\right)$ and let $x_{2}=\left(x_{I, 2}, x_{Q, 2}\right) \neq x_{1}$ be one of the possibly multiple nearest symbols to $x_{1}$ in terms of distance between its in-phase component, i.e., $\left|x_{I, 2}-x_{I, 1}\right|$; this distance can be written as:

$$
\left|x_{I, 1}-x_{I, 2}\right|=2\left|N\left(p_{I, 1}-p_{I, 2}\right)-\left(p_{Q, 1}-p_{Q, 2}\right)\right| \sin \alpha .
$$

Assume that $\left|x_{I, 2}-x_{I, 1}\right|<2 \sin \alpha$; according to (22): $\left|N\left(p_{I, 1}-p_{I, 2}\right)+\left(p_{Q, 2}-p_{Q, 1}\right)\right|<1$; then $\left(p_{I, 1}, p_{Q, 1}\right)=$ $\left(p_{I, 2}, p_{Q, 2}\right)$, which contradicts the condition $x_{2} \neq x_{1}$. Furthermore, assume that $\left|x_{I, 2}-x_{I, 1}\right|>2 \sin \alpha$. Let us define $x_{3}$ a rotated symbol corresponding to $\left(p_{I, 3}, p_{Q, 3}\right)=\left(p_{I, 1}, p_{Q, 1}-1\right)$ if $p_{Q, 1} \neq 0$, and to $\left(p_{I, 3}, p_{Q, 3}\right)=\left(p_{I, 1}, p_{Q, 1}+1\right)$ otherwise. According to (22): $\left|x_{I, 3}-x_{I, 1}\right|=2 \sin \alpha$, which contradicts the condition that $x_{2}$ is one of the nearest symbol to $x_{1}$ in terms of the distance $\left|x_{I, 2}-x_{I, 1}\right|$. Therefore, for any rotated symbol, its nearest neighbor projected on the in-phase component is at the distance $d_{1 \mathrm{D}, \min }=2 \sin \alpha$. Similarly, we can prove property 1 for the quadrature components.

We can observe property 1 for the 4-QAM constellation in Fig. 2. Thus, in the sequel, the modulation corresponding to the choice of the rotation angle $\alpha=\arctan (1 / N)$ is referred to as uniformly projected rotated and cyclic Q delay (UP-RCQD) QAM.

Property 2. For the UP-RCQD $M-Q A M$, the integer index pair $\left(p_{I}, p_{Q}\right)$ can be uniquely determined by a single rotated component $x_{m}$ where $m$ is in $\{I, Q\}$.

Proof: Let us define the integers $T_{I}$ and $T_{Q}$ as follows:

$$
\begin{aligned}
T_{I} & =\frac{x_{I}}{2 \sin \alpha}+\frac{(M-1)}{2}=N p_{I}+\left(-p_{Q}+(N-1)\right), \\
T_{Q} & =\frac{x_{Q}}{2 \sin \alpha}+\frac{(M-1)}{2}=N p_{Q}+p_{I} .
\end{aligned}
$$

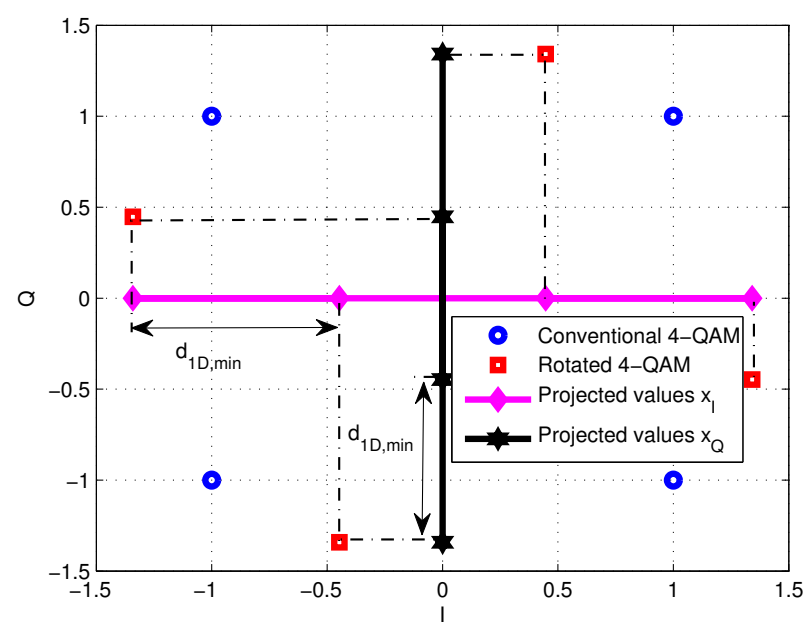

Figure 2. Uniformly projected RCQD 4-QAM signal.

Equation (23) (resp. (24)) indicates that $p_{I}$ (resp. $p_{Q}$ ) is carried on the $\left(\log _{2} M\right) / 2$ most significant bits of the binary representation of the integer $T_{I}$ (resp. $T_{Q}$ ), whereas $p_{Q}$ (resp. $\left.p_{I}\right)$ is carried on its $\left(\log _{2} M\right) / 2$ least significant bits. Thus from the value taken by $T_{I}, p_{I}$ and $p_{Q}$ can be obtained as:

$$
\left\{\begin{array}{l}
p_{I}=\left\lfloor\frac{T_{I}}{N}\right\rfloor, \\
p_{Q}=N-1-\left(T_{I}-N p_{I}\right),
\end{array}\right.
$$

where $\lfloor a\rfloor$ denotes the integer part of $a$.

Similarly, from the value taken by $T_{Q}, p_{I}$ and $p_{Q}$ can be obtained as:

$$
\left\{\begin{array}{l}
p_{Q}=\left\lfloor\frac{T_{Q}}{N}\right\rfloor \\
p_{I}=T_{Q}-N p_{Q}
\end{array}\right.
$$

Therefore, Property 2 is proved.

In practice, all the divide and multiply operations by $N$ can be implemented by $\left(\log _{2} M\right) / 2$ right-shifting or left-shifting. Therefore, neither division nor multiplication is needed. Moreover, since the relation between the pairs $\left(T_{I}, T_{Q}\right)$ and $\left(p_{I}, p_{Q}\right)$ is fixed for a given constellation, the mutual conversion can be implemented by a look-up table which can reduce the computational complexity.

Property 3. For the UP-RCQD M-QAM constellation, any $N$ consecutive values of $T_{I}$ (resp. $T_{Q}$ ) cover the $N$ possible values of $p_{Q}$ (resp. $\left.p_{I}\right)$.

Proof: $T_{I}$ and $T_{Q}$ take integer values; hence the remainders of the division of any $N$ consecutive values of $T_{I}$ (resp. $T_{Q}$ ) by $N$ are $0,1, \cdots, N-1$ and there is a one to one correspondence with $p_{Q}$ (resp. $p_{I}$ ) according to (25) (resp. (26)). Therefore, Property 3 is proved. 


\section{B. UP-RCQD Demapping Solution}

From (8) and (9), the received components $r_{m}(m \in\{I, Q\})$ can be equalized as follow for nonzero $h_{m}$ :

$$
\begin{aligned}
R_{m} & =\frac{r_{m}}{2 \beta h_{m} \sin \alpha}+\frac{(M-1)}{2} \\
& =\frac{x_{m}}{2 \sin \alpha}+\frac{(M-1)}{2}+\frac{v_{m}}{2 \beta h_{m} \sin \alpha} \\
& =T_{m}+\frac{v_{m}}{2 \beta h_{m} \sin \alpha},
\end{aligned}
$$

where $T_{m}$ is given by (23) or (24).

Therefore, the term $\left|r_{m}-h_{m} \beta x_{m}\right|^{2}$ can be modified as:

$$
\left|r_{m}-h_{m} \beta x_{m}\right|^{2}=(2 \beta \sin \alpha)^{2}\left|h_{m}\left(R_{m}-T_{m}\right)\right|^{2},
$$

where $m$ is in $\{I, Q\}$ and $R_{m}$ can be considered as the equivalent equalized noisy observation point of the $\mathrm{I}$ or $\mathrm{Q}$ component.

1) LLR Computation in the Integer-Domain: With (28), the Max-Log LLR computation (15) becomes:

$$
\begin{aligned}
\Lambda\left(l^{i}(x)\right)= & \frac{(2 \beta \sin \alpha)^{2}}{\sigma^{2}} \min _{T \in \mathbf{T}_{1}^{i}}\left\{\left|h_{I}\left(R_{I}-T_{I}\right)\right|^{2}+\left|h_{Q}\left(R_{Q}-T_{Q}\right)\right|^{2}\right\} \\
& -\frac{(2 \beta \sin \alpha)^{2}}{\sigma^{2}} \min _{T \in \mathbf{T}_{0}^{i}}\left\{\left|h_{I}\left(R_{I}-T_{I}\right)\right|^{2}+\left|h_{Q}\left(R_{Q}-T_{Q}\right)\right|^{2}\right\},
\end{aligned}
$$

where $\mathbf{T}_{0}^{i}$ (resp. $\mathbf{T}_{1}^{i}$ ) denotes the set of the constellation points whose i-th bit is equal to 0 (resp. 1).

If an erasure event occurs over one of the components, (29) is simplified as:

$$
\begin{aligned}
\Lambda\left(l^{i}(x)\right) & =\frac{2(2 \beta \sin \alpha)^{2}}{\sigma^{2}} \min _{T \in \mathbf{T}_{1}^{i}}\left\{\left|h_{m}\left(R_{m}-T_{m}\right)\right|^{2}\right\} \\
& -\frac{2(2 \beta \sin \alpha)^{2}}{\sigma^{2}} \min _{T \in \mathbf{T}_{0}^{i}}\left\{\left|h_{m}\left(R_{m}-T_{m}\right)\right|^{2}\right\},
\end{aligned}
$$

where $m$ can either be I or $\mathbf{Q}$ according to the remaining nonerased component.

For the fading channel, the Max-Log demapper in (29) needs the calculus of $2 \log _{2} M$ minimal Euclidean distance terms like $\min _{T \in \mathbf{T}_{b}^{i}}\left\{\left|h_{I}\left(R_{I}-T_{I}\right)\right|^{2}+\left|h_{Q}\left(R_{Q}-T_{Q}\right)\right|^{2}\right\}$ to compute $\log _{2} M$ LLR values. Among these distances, $\log _{2} M$ distance terms can be found once the maximum likelihood (ML) point corresponding to $T_{o p t}=\left(T_{I, o p t}, T_{Q, o p t}\right)$, i.e., the nearest constellation point to the received observation, is located, while the other $\log _{2} M$ terms in (29) requires to find the closest constellation point (to the received point) $T_{c p l m}^{i}=\left(T_{I, c p l m}^{i}, T_{Q, c p l m}^{i}\right)$ whose i-th information bit is complementary to the $\mathrm{i}$-th bit of the global optimum $T_{\text {opt }}$. Therefore, the proposed demapper is divided into two steps: locating the global optimum point and finding complementary points. In summary, (29) can be written as:

$\Lambda\left(l^{i}(x)\right)= \begin{cases}\frac{(2 \beta \sin \alpha)^{2}}{\sigma^{2}}\left(d\left(T_{c p l m}^{i}, R\right)-d\left(T_{o p t}, R\right)\right), & l^{i}\left(T_{o p t}\right)=0, \\ \frac{(2 \beta \sin \alpha)^{2}}{\sigma^{2}}\left(d\left(T_{o p t}, R\right)-d\left(T_{c p l m}^{i}, R\right)\right), & l^{i}\left(T_{o p t}\right)=1,\end{cases}$ where $d\left(T_{o p t}, R\right)$ and $d\left(T_{c p l m}^{i}, R\right)$ denote the 2D Euclidean distances between the point $T_{x}\left(T_{o p t}\right.$ or $\left.T_{c p l m}^{i}\right)$ and the received point $\mathrm{R}$ with:

$$
d\left(T_{x}, R\right)=\left|h_{I}\left(R_{I}-T_{I, x}\right)\right|^{2}+\left|h_{Q}\left(R_{Q}-T_{Q, x}\right)\right|^{2} .
$$

For the fading channel with erasure, the Max-Log demapper in (30) is similar to (31):

$\Lambda\left(l^{i}(x)\right)=\left\{\begin{array}{l}\frac{2(2 \beta \sin \alpha)^{2}}{\sigma^{2}}\left(d\left(T_{c p l m}^{i}, R_{m}\right)-d\left(T_{o p t}, R_{m}\right)\right), l^{i}\left(T_{o p t}\right)=0, \\ \frac{2(2 \beta \sin \alpha)^{2}}{\sigma^{2}}\left(d\left(T_{o p t}, R_{m}\right)-d\left(T_{c p l m}^{i}, R_{m}\right)\right), l^{i}\left(T_{o p t}\right)=1,\end{array}\right.$

where $m$ can either be I or $\mathrm{Q}$ according to the remaining nonerased component; $d\left(T_{o p t}, R_{m}\right)$ and $d\left(T_{c p l m}^{i}, R_{m}\right)$ denote the 1D Euclidean distances between the remaining component $m$ of the point $T_{x}\left(T_{o p t}\right.$ or $\left.T_{c p l m}^{i}\right)$ and the received observation $R_{m}$, which are given by:

$$
d\left(T_{x}, R_{m}\right)=\left|h_{m}\left(R_{m}-T_{m, x}\right)\right|^{2} .
$$

In order to simplify the notation, in the sequel, when one component (I or Q) is erased, $T_{\text {opt }}$ (resp. $T_{\text {cplm }}^{i}$ ) is no more a $2 \mathrm{D}$ point and we rather consider instead that $T_{o p t}=T_{m, o p t}$ (resp. $T_{c p l m}^{i}=T_{m, c p l m}^{i}$ ), where $m$ can either be $\mathrm{I}$ or $\mathrm{Q}$ according to the remaining non-erased component.

\section{2) Global Optimum:}

Proposition. For the UP-RCQD M-QAM constellation, the local optimum $T_{m, \text { Loc opt }}$ with $m$ being either $I$ or $Q$ i.e., the nearest point to the equalized observation $R_{m}$ is found by minimizing (28) as follows:

$$
T_{m, \text { Loc opt }}= \begin{cases}0, & \text { if } R_{m} \leq 0, \\ \operatorname{round}\left(R_{m}\right), & \text { if } 0 \leq R_{m}<(M-1), \\ M-1, & \text { if } R_{m} \geq(M-1) .\end{cases}
$$

This Proposition indicates that each observation $R_{m}$ leads to a unique 1D local nearest point; if reciprocally $T_{I, L o c ~ o p t}$ and $T_{Q, \text { Loc opt }}$ given by (35) lead to the same pair $\left(p_{I}, p_{Q}\right)$ (see (25)-(26)), then one necessarily finds the global optimum estimate because the Euclidean distance terms (28) are both minimized (refer to (29)). However, in poor channel conditions, the two local optimums $T_{I, \text { Loc opt }}$ and $T_{Q, \text { Loc opt }}$ may lead to different pairs $\left(p_{I}, p_{Q}\right)$ and the global optimum point minimizing (29) may not coincide with either of those two pairs. In this case and in order to locate the global optimum, we propose to search $T_{\text {opt }}$ in regions around $R_{m}$ with radius $d$ with $m$ in $\{I, Q\}$. These regions can be written as:

$$
\widetilde{\mathbf{T}}_{m}= \begin{cases}\{0, \cdots, 2 d-1\}, & \text { if } R_{m}<d, \\ \{M-2 d, \cdots, M-1\}, & \text { if } R_{m} \geq M-d, \\ \left\{\left\lfloor R_{m}\right\rfloor-d+1, \cdots,\left\lfloor R_{m}\right\rfloor+d\right\}, & \text { otherwise. }\end{cases}
$$

Each point $T$ within $\widetilde{\mathbf{T}}_{m}$ uniquely determines a pair $\left(p_{I}, p_{Q}\right)$ and this pair uniquely locates a non-rotated constellation point $\left(s_{I}, s_{Q}\right)$ candidate. Therefore, $\widetilde{\mathbf{T}}_{m}$ has exactly $2 d$ candidate points and the probability to find the true global optimum point 
$T_{\text {opt }}$ within the $4 d$ points of the sets $\widetilde{\mathbf{T}}_{I}$ and $\widetilde{\mathbf{T}}_{Q}$ increases with the radius $d$.

Furthermore, differently from the sphere decoding proposed in [24], $R_{m}$ in (36) is the equalized observation, whereas [24] uses the received signal $r_{m}$. Moreover, the integer diameter $2 \mathrm{~d}$ represents the number of points that are exactly found within this sphere, whereas the radius in [24] does not guarantee to find points involved in the demapping, and it is even unable to guarantee that there will be at least one point involved.

If an erasure event occurs on one component, it should be noted that the local optimum point $T_{m, L o c ~ o p t}$ is necessarily the global optimum $T_{\text {opt }}$ (refer to (28) and (30)).

3) Complementary Candidate Points for the LLR Computation for Fading Channels: According to Property 3, the region centered around the local optimum $T_{I, L o c ~ o p t}$ (resp. $\left.T_{Q, \text { Loc opt }}\right)$ with radius $d=N / 2$ contains all the possible values of $p_{Q}$ (resp. $p_{I}$ ). Therefore, $\widetilde{\mathbf{T}}_{I}$ and $\widetilde{\mathbf{T}}_{Q}$ guarantee to find a point $T_{c p l m}^{i}$ for any bit index $i$ corresponding to the complementary of $T_{I, \text { Loc opt }}$ or $T_{Q, \text { Loc opt }}$ considered bit.

4) Complementary Candidate Points for the LLR Computation for Fading Erasure Channels: For the fading channel with erasure, we can simplify even more the complexity of the demapper for the Gray mapped UP-RCQD $M$-QAM, without compromising its performance when compared to the MaxLog demapper thanks to the two following properties.

Let us define $\mathbf{T}_{I}$ (resp. $\mathbf{T}_{Q}$ as the set of all possible in-phase components $T_{I}$ (resp. quadrature components $T_{Q}$ ): $\mathbf{T}_{m}=\left\{T_{m, 0}, T_{m, 1}, \cdots, T_{m, M-1}\right\}$, where $T_{m, 0}<T_{m, 1}<$ $\cdots<T_{m, M-1}$ and $m$ is in $\{I, Q\}$.

Lemma 1. For the Gray mapped UP-RCQD M-QAM constellation, let $d_{1}$ and $d_{2}$ be two integers such that $0<d_{1}<$ $d_{2}<M-1$. If $l^{i}\left(T_{m, d_{1}}\right)=l^{i}\left(T_{m, d_{2}}\right)=b$ where $b$ is in $\{0,1\}$ and $i \in\left\{0,1, \cdots, \log _{2}(M)-1\right\}$, and if $l^{i}\left(T_{m, k}\right)=\bar{b}$ for all integers $k$ spanning from $d_{1}+1$ to $d_{2}-1$, then $d_{2}-d_{1}$ is odd.

This lemma directly comes from the reflection characteristic of the Gray mapping (see e.g. the illustration for the 4 bits of the 16-QAM signal in Fig. 3, where one can see that, except at the edges, each bit is generally constant over an even number of adjacent bits); note that Lemma 1 is not concerned by $T_{m, 0}$ and $T_{m, M-1}$ which are different from the other points because they are at opposite edges.

Property 4. For the Gray-mapped UP-RCQD M-QAM constellation, let us consider the only non-erased component $T_{m}$ of a signal $T=\left(T_{I}, T_{Q}\right)$ whose $i$-th bit is b. There is only one point at the smallest distance of $T_{m}$ whose $i$-th bit is equal to the binary complementary $\bar{b}$.

Proof: Assume that Property 4 is false and that there are two different component points $T_{m, c_{1}}$ and $T_{m, c_{2}}$ at the same minimum distance of $T_{m}$ with $i^{\text {th }}$ bit equal to $\bar{b}$, i.e.:

$$
\begin{aligned}
\left|T_{m}-T_{m, c_{1}}\right| & =\left|T_{m}-T_{m, c_{2}}\right| \\
& =\min _{T_{m, k} \in \mathbf{T}_{m} \mid l^{i}\left(T_{m, k}\right)=\bar{b}}\left|T_{m}-T_{m, k}\right| .
\end{aligned}
$$

This means that there is an odd number of consecutive points with $\mathrm{i}$-th bit equal to $b$ between $T_{m, c_{1}}$ and $T_{m, c_{2}}$;

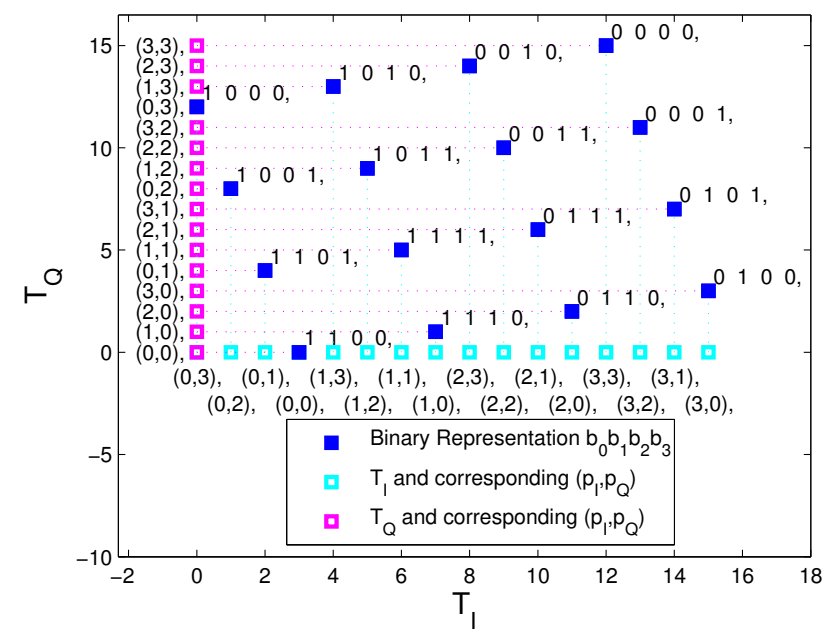

Figure 3. $\left(T_{I}, T_{Q}\right)$ and the corresponding binary representation $\left(b_{0} b_{1} b_{2} b_{3}\right)$ of the 16-QAM signal.

then, according to Lemma $1, T_{m}$ can only be either $T_{m, 0}$ or $T_{m, M-1}$; in both cases, $T_{m}$ cannot be located between $T_{m, c_{1}}$ and $T_{m, c_{2}}$ since $T_{m}$ is an edge point. Therefore, this contradiction proves Property 4.

Lemma 2. If $a, b$ and $c$ are real numbers such that $|a-b| \geq$ $|b-c|$, then:

$|a-c|= \begin{cases}|a-b|+|b-c|, & \text { if } \min (a, c) \leq b \leq \max (a, c), \\ |a-b|-|b-c|, & \text { otherwise. }\end{cases}$

This Lemma is needed to prove the following Property.

Property 5. Consider, for the Gray-mapped UP-RCQD $M$ $Q A M$ constellation, the only non erased component $R_{m}$ and let $T_{\text {opt }}$ be the global optimum point whose $i$-th bit is $b$. If $T_{m, c p 2}^{i}$ is the nearest point to $T_{\text {opt }}$ with $i$-th bit equal to $\bar{b}$ :

$$
T_{m, c p 2}^{i}=\underset{T_{m, k} \in \mathbf{T}_{m} \mid l^{i}\left(T_{m, k}\right)=\bar{b}}{\operatorname{argmin}}\left|T_{o p t}-T_{m, k}\right|,
$$

and if $T_{c p l m}^{i}$ is the nearest point to $R_{m}$ with $i$-th bit equal to $\bar{b}$ :

$$
T_{c p l m}^{i}=\underset{T_{m, k} \in \mathbf{T}_{m} \mid l^{i}\left(T_{m, k}\right)=\bar{b}}{\operatorname{argmin}}\left|R_{m}-T_{m, k}\right|,
$$

then $T_{m, c p 2}^{i}$ is equal to $T_{c p l m}^{i}$.

Proof: Consider $T_{m}^{i, \bar{b}} \in \mathbf{T}_{m}$ whose i-th bit is $\bar{b}$.

If $R_{m} \leq 0$ or $R_{m} \geq M-1$ then $\min \left(R_{m}, T_{m}^{i, \bar{b}}\right) \leq T_{\text {opt }} \leq$ $\max \left(R_{m}, T_{m}^{i, \bar{b}}\right)$ and according to lemma 2 we have:

$$
\left|R_{m}-T_{m}^{i, \bar{b}}\right|=\left|R_{m}-T_{o p t}\right|+\left|T_{o p t}-T_{m}^{i, \bar{b}}\right|,
$$

so that:

$$
\begin{aligned}
\min _{T_{m, k} \in \mathbf{T}_{m} \mid l^{i}\left(T_{m, k}\right)=\bar{b}}\left|R_{m, k}-T_{m, k}\right| & =\left|R_{m}-T_{o p t}\right| \\
& +\operatorname{Tin}_{T_{m, k} \in \mathbf{T}_{m} \mid l^{i}\left(T_{m, k}\right)=\bar{b}}\left|T_{o p t}-T_{m, k}\right|,
\end{aligned}
$$


thus $T_{m, c p 2}^{i}=T_{c p l m}^{i}$ for $R_{m} \leq 0$ or $R_{m} \geq M-1$.

If $0<R_{m}<M-1$, according to the triangular inequality, the distance between $R_{m}$ and $T_{m, c p 2}^{i}$ can be upper bounded as:

$$
\begin{aligned}
\left|R_{m}-T_{m, c p 2}^{i}\right| & =\left|R_{m}-T_{o p t}+T_{o p t}-T_{m, c p 2}^{i}\right| \\
& \leq\left|R_{m}-T_{o p t}\right|+\left|T_{o p t}-T_{m, c p 2}^{i}\right| \\
& \leq 0.5+\min _{T_{m, k} \in \mathbf{T}_{m} \mid l^{i}\left(T_{m, k}\right)=\bar{b}}\left|T_{o p t}-T_{m, k}\right| .
\end{aligned}
$$

Consider another point $T_{m}^{i, \bar{b}} \neq T_{m, c p 2}^{i}$ whose $\mathrm{i}$-th bit is $\bar{b}$; the distance between $R_{m}$ and $T_{m}^{i, \bar{b}}$ can be lower bounded:

$$
\begin{aligned}
& \left|R_{m}-T_{m}^{i, \bar{b}}\right|=\left|T_{o p t}-T_{m}^{i, \bar{b}}+R_{m}-T_{o p t}\right| \\
& \geq\left|T_{o p t}-T_{m}^{i, \bar{b}}\right|-\left|R_{m}-T_{o p t}\right| \\
& \geq\left(\min _{T_{m, k} \in \mathbf{T}_{m} \mid l^{i}\left(T_{m, k}\right)=\bar{b}}\left|T_{o p t}-T_{m, k}\right|+1\right)-0.5,
\end{aligned}
$$

where the second inequality in (44) comes from Property 4. From (44) and then (43), one can get the following inequality for any $T_{m}^{i, \bar{b}}$ :

$$
\begin{aligned}
\left|R_{m}-T_{m}^{i, \bar{b}}\right| & \geq 0.5+\underset{T_{m, k} \in \mathbf{T}_{m} \mid l^{i}\left(T_{m, k}\right) \bar{b}}{\min }\left|T_{o p t}-T_{m, k}\right| \\
& \geq\left|R_{m}-T_{m, c p 2}^{i}\right| .
\end{aligned}
$$

Therefore, taking the minimum argument on the left side of (45), we find that $T_{c p l m}^{i}=T_{m, c p 2}^{i}$ for $0<R_{m}<M-1$ (see (40)). This completes the proof of Property 5.

Property 5 states that all the complementary points $T_{c p l m}^{i}$ nearest to the received observation can be located using the global optimum $T_{\text {opt }}$ instead of the equalized observation $R_{m}$. Thus, once $R_{m}$ is rounded to $T_{o p t}$, the points involved in the LLR computations are fixed and there is no more need for any Euclidean distance comparison which reduces considerably the computation for the erasure channel.

We can now detail how to systematically find for any bit index, the point having a complementary bit to $T_{\text {opt }}$.

When only the Q component is erased, from (23), the distance between the global optimum $T_{o p t}$ and another point $T_{I}\left(T_{I} \neq T_{\text {opt }}\right)$ is given by:

$$
\left|T_{I, o p t}-T_{I}\right|=\left|N\left(p_{I, o p t}-p_{I}\right)-\left(p_{Q, o p t}-p_{Q}\right)\right| .
$$

Since, from the system model of section II.A, odd-indexed bits only depend of $p_{Q}$, the nearest points $T_{I}$ with complementary odd bits to $T_{I, \text { opt }}$ can directly be found among the nearest $N / 2$ points to $T_{I, \text { opt }}$. To find the complementary points for even-indexed bits, we need the nearest $N / 2$ points to $p_{I, o p t}$; from (46), they cannot be found among the neighbors of $T_{I, \text { opt }}$, thus we need to set for each of them a fixed $p_{Q}$ value; in order to find $T_{c p l m}^{i}$ for even $i$ values, we set $p_{Q}=0$ (resp. $\left.p_{Q}=N-1\right)$ if $\left(p_{I, \text { opt }}-p_{I}\right)>0\left(\right.$ resp. $\left.\left(p_{I, \text { opt }}-p_{I}\right)<0\right)$.

Similarly, when only the I component is erased, from (24), the distance between the global optimum $T_{o p t}$ and another point $T_{Q}\left(T_{Q} \neq T_{o p t}\right)$ is given by:

$$
\left|T_{Q, o p t}-T_{Q}\right|=\left|N\left(p_{Q, o p t}-p_{Q}\right)-\left(p_{I, o p t}-p_{I}\right)\right| .
$$

Since even-indexed bits only depend of $p_{I}$, the nearest points $T_{Q}$ with complementary even bits to $T_{Q \text {,opt }}$ can directly be found among the nearest $N / 2$ points to $T_{Q, o p t}$. To find the complementary points for odd-indexed bits, we need the nearest $N / 2$ points to $p_{Q, o p t}$. They cannot be found among the neighbors of $T_{Q, o p t}$, thus we need to set for each of them a fixed $p_{I}$ value; in order to find $T_{c p l m}^{i}$ for odd $i$ values, we set $p_{I}=0$ (resp. $\left.p_{I}=N-1\right)$ if $\left(p_{Q, o p t}-p_{Q}\right)>0$ (resp. $\left.\left(p_{Q, o p t}-p_{Q}\right)<0\right)$.

\section{The Proposed Sphere-based Demapping Algorithm}

Gathering the results of the previous sections, we now propose a synthesis of the sphere-based algorithm which can be decomposed into the following four steps:

1) Transform the received components $r_{m}$ into $R_{m}$ by using (27). If one component is erased, $R_{m}$ is set to 0 . If both components (I/Q) are erased, the LLR values are directly set to 0 .

2) For fading channels without erasure, obtain $N$ points centered on $R_{I}$ and $N$ points centered on $R_{Q}$ using (36) with $d=N / 2$, and find within the $2 N$ points the global optimum at minimum distance from $R$ calculated with (32). Note that if the two local optimum points lead to the same $\left(p_{I}, p_{Q}\right)$, one directly obtain the ML estimate $T_{\text {opt }}$. If only one component remains in the case of an erasure channel, (35) is used to locate directly the global optimum, and at last, the minimum distance is computed with (34).

3) For the non-erasure case, all the points with bit complementary to those of $T_{o p t}$ are selected among the $2 N$ points for which the minimum distance is calculated with (32). For the erasure channel, when only the in-phase (resp. quadrature) component is erased all the $N$ points are selected using the rules below (46) (resp. 47); the minimum distances for the complementary points are then calculated with (34).

4) Once the Euclidean distances are obtained for $T_{o p t}$ in step 2 and for $T_{c p l m}^{i}$ in step 3, the LLR computation can be performed for all bits by using (31) (resp. (33)) for the classical fading (resp. fading erasure) channel.

\section{Complexity Analysis}

The detailed analysis on the complexity of the algorithm is made and evaluated in terms of Candidate Points (CP), Real Multiplications (RM), Real Comparisons (RC), Real Inversions (RI) and Real Sums (RS), where a RS can either designate a real addition or a real subtraction.

With a conventional rotation angle $\alpha$ at the transmitter side, the components $x_{I}$ and $x_{Q}$ of the rotated $M$-QAM signal are obtained with equation (4). It can be observed that a rotation operation requires $4 \mathrm{RMs}$ and 2 RSs. However, when $\alpha=$ $\arctan (1 / N)$, the corresponding components are obtained by (18) and (19). As previously mentioned, the multiplication by a factor $N$ can be implemented by $\left(\log _{2} M\right) / 2$ left-shifts. Therefore the rotation operation in this case requires $2 \mathrm{RMs}$, 2 RSs and 2 left-shifts.

At the receiver side, for the fading channel without any erasure: 
- In step 1 of section III. $C,\left(1 / 2 \beta h_{m} \sin \alpha\right)$ and $((M-$ 1)/2) are known constant terms; therefore the transformation (27) of $r_{I}$ into $R_{I}$ and $r_{Q}$ into $R_{Q}$ need globally 2 RSs, 2 RMs and 2 RIs.

- In step 2, choosing in (36) the kind of intervals for $R_{I}$ and $R_{Q}$ requires 4 RCs. Each term $\left|h_{I}\left(R_{I}-T_{I}\right)\right|^{2}+$ $\left|h_{Q}\left(R_{Q}-T_{Q}\right)\right|^{2}$ takes 4 RMs and 3 RSs. Since there are $2 N$ distances to compute, $8 N$ RMs and $6 N$ RSs are globally required. Searching the point with minimum distance needs $(2 N-1)$ RCs. Therefore, step 2 requires a total of $8 N$ RMs, $6 N$ RSs and $(2 N+3)$ RCs.

- In step 3, there are $\log _{2} M$ minimum distances required for $\log _{2} M$ complementary bits. Since each bit needs $(2 N-2)$ RCs, this step takes a total of $(2 N-2) \log _{2} M$ RCs.

- In step 4, multiplying the term $\left((2 \beta \sin \alpha)^{2} / \sigma^{2}\right)$ requires $1 \mathrm{RM}$. Thus, there is a total of $\log _{2} M$ RMs and $\log _{2} M$ RSs for the $\log _{2} M$ bits.

Therefore, performing LLR computations of a symbol in (31) globally requires $2 N$ CPs, $\left(8 N+\log _{2} M+2\right)$ RMs, $\left((2 N-2)\left(1+\log _{2} M\right)+5\right)$ RCs, 2 RIs and $\left(6 N+\log _{2} M+\right.$ 2) RSs.

For the case with one erased component:

- In step 1, the transformation of $R_{m}$ requires $1 \mathrm{RS}, 1 \mathrm{RM}$ and $1 \mathrm{RI}$.

- In step 2, rounding the term $R_{m}$ to $T_{m, o p t}$ needs 2 RCs. Computing the distance term $\left|h_{m}\left(R_{m}-T_{m, o p t}\right)\right|^{2}$ takes 2 RMs and 1 RS. Therefore, there is a total of 2 RMs, 1 RS and 2 RCs.

- In step 3, selecting two CP regions $(N / 2$ CPs belong to each region) necessitates $4 \mathrm{RCs}$. Those $N$ CPs require $2 N$ RMs and $N$ RSs to compute $N$ distance terms. Since there are $\log _{2} M$ minimum distances required for $\log _{2} M$ complementary bits and since each of them needs $(\mathrm{N} / 2-$ 1) RCs, this step needs a total of $2 N$ RMs, $N$ RSs and $\left((N / 2-1) \log _{2} M+4\right)$ RCs.

- In step 4 , multiplying the term $\left(2(2 \beta \sin \alpha)^{2} / \sigma^{2}\right)$ requires $1 \mathrm{RM}$. Thus, there is a total of $\log _{2} M$ RMs and $\log _{2} M$ RSs for the $\log _{2} M$ bits.

Therefore, performing LLR computations of a symbol in (33) globally requires $N$ CPs, $\left(2 N+\log _{2} M+3\right)$ RMs, $\left((N / 2-1) \log _{2} M+4\right)$ RCs, 1 RI and $\left(N+\log _{2} M+4\right)$ RSs.

\section{Numerical Results}

This section is divided into two parts. The first part discusses the performance of the UP-RCQD in terms of bit-interleaved coded-modulation (BICM) capacity [26] and bit error rate (BER) over fading channels with and without erasure events. The second part compares the proposed sphere demapping algorithm with other published algorithms for the 256-QAM signal in terms of complexity and performance, where the 256-QAM is the only constellation using the rotation angle $\alpha=\arctan (1 / N)$ in DVB-T2; it thus enables some direct performance and complexity comparisons.

Note that the Rayleigh fading channel models with and without erasure events are defined in [25].

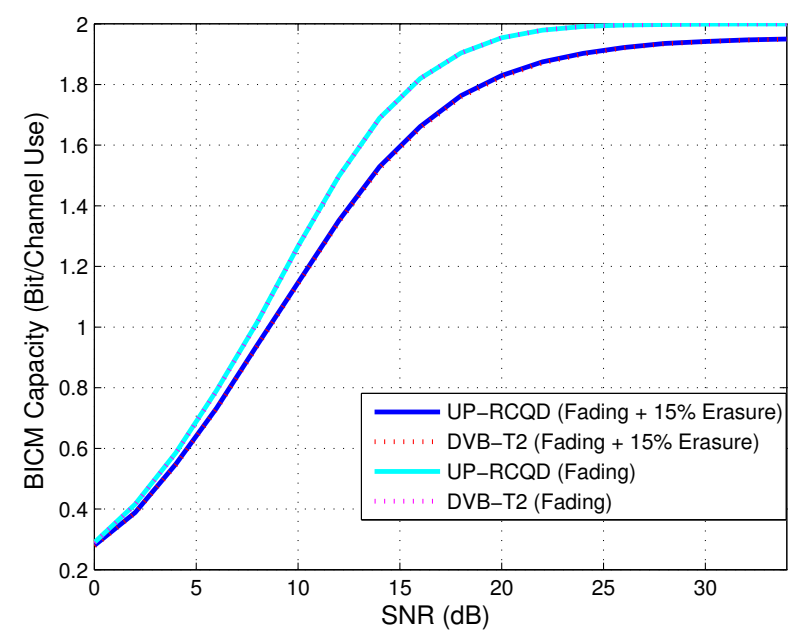

Figure 4. RCQD QPSK BICM capacity comparison between the UP-RCQD solution and the DVB-T2 angle.

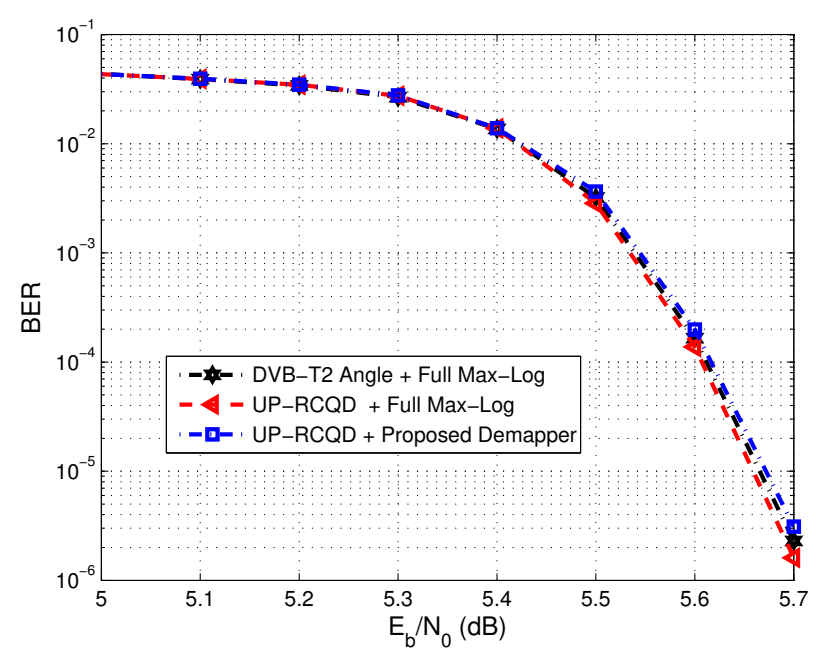

Figure 5. BER comparison between the UP-RCQD solution and the DVB-T2 angle for QPSK signals over Rayleigh fading channels.

\section{A. The UP-RCQD Solution}

In these simulations, BICM capacities are evaluated through Monte-Carlo simulations and BERs are first obtained with the DVB-T2 64800-bit long LDPC code of rate 4/5 with a 25iteration min-sum decoder.

Figs. 4, 7 and 10 compare the BICM capacity performance between the UP-RCQD solution and the DVB-T2 rotation angles for QPSK, 16-QAM and 64-QAM signals over the fading channel with and without erasure events, whereas Figs. 5, 8 and 11 (resp. Figs. 6, 9 and 12) compare their corresponding BER performance over Rayleigh fading channels without erasure (resp. with $15 \%$ of erasure events).

It can first be observed that for any constellation the BICM capacity of the UP-RCQD is about the same as the BICM capacity performance of the DVB-T2 angle over the fading channel without any erasure. This indicates that similar BER performance can theoretically be achieved for both cases over 


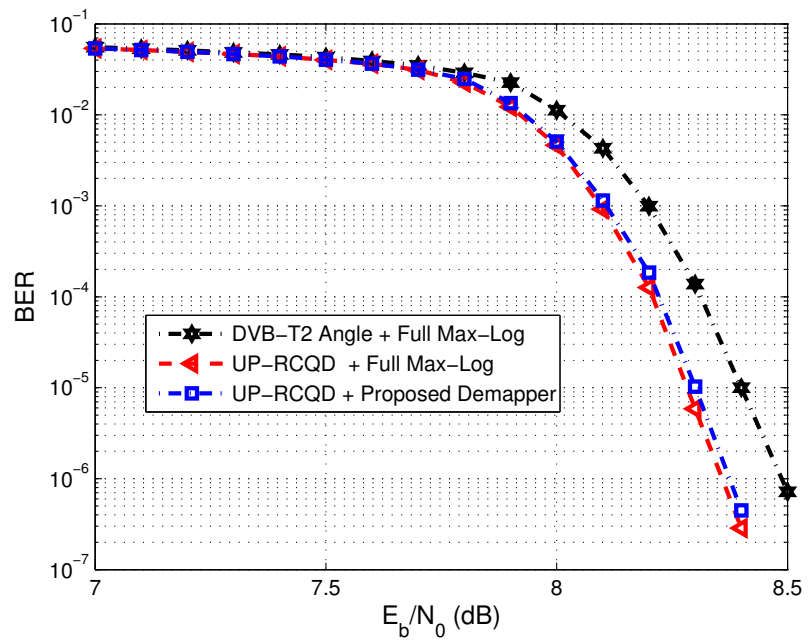

Figure 6. BER comparison between the UP-RCQD solution and the DVB-T2 angle for QPSK signals over Rayleigh fading channels with 15\% of erasure events.

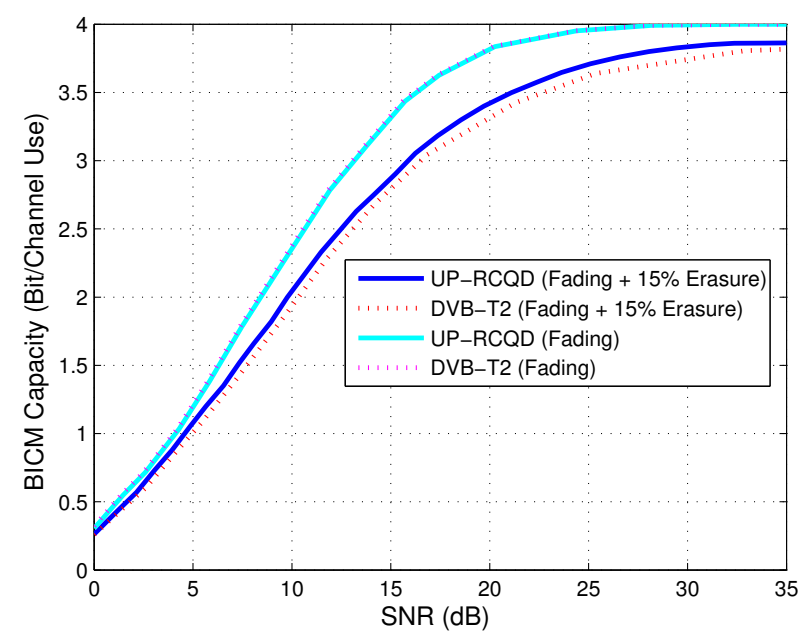

Figure 7. RCQD 16-QAM BICM capacity comparison between the UPRCQD solution and the DVB-T2 angle.

such fading channels; Fig. 5, Fig. 8 and Fig. 11 confirm this expectation. Furthermore, these figures also indicate that for the UP-RCQD solution, the proposed demapper achieves almost the same performance as the more complex Max-Log algorithm.

The UP-RCQD solution achieves a better BICM capacity compared to the DVB-T2 solution over the fading channel with $15 \%$ of erasure events (see Figs. 4, 7 and 10). This leads to the result that the considered rotation angle outperforms the original DVB-T2 rotation angle by $0.1 \mathrm{~dB}, 0.5 \mathrm{~dB}$ and $0.75 \mathrm{~dB}$ for the QPSK, 16-QAM and 64-QAM signal, respectively, at $B E R=10^{-6}$ (see Figs. 6, 9 and 12). It is worth mentioning that the coding gain depends on both the constellation size and the code rate (CR). Figs. 13, 14 and 15 compare the BER obtained with the UP-RCQD solution and the DVB-T2 angles for the DVB-T2 LDPC codes with code rates $\mathrm{CR}=1 / 2$ and $\mathrm{CR}=5 / 6$ over a Rayleigh fading channel with $15 \%$ of

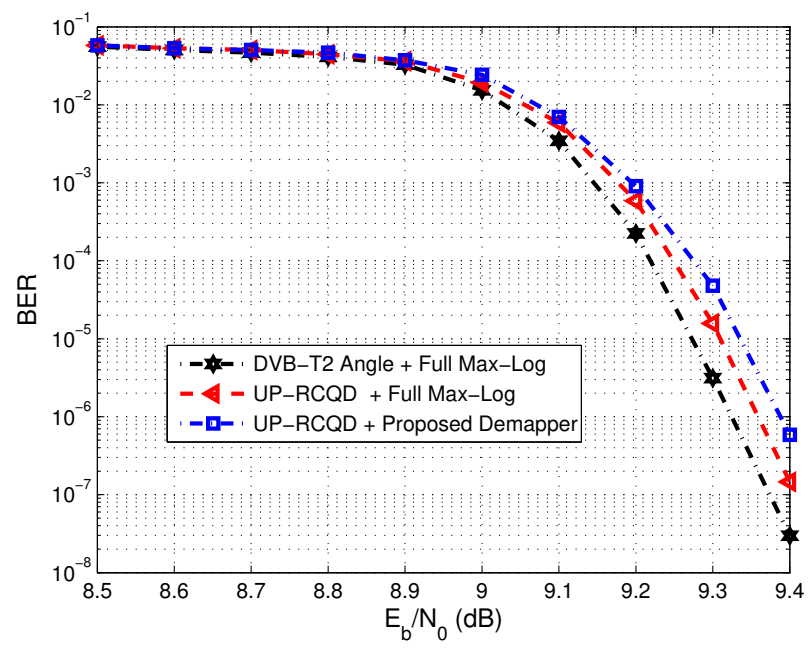

Figure 8. BER comparison between the UP-RCQD solution and the DVB-T2 angle for 16-QAM signals over Rayleigh fading channels.

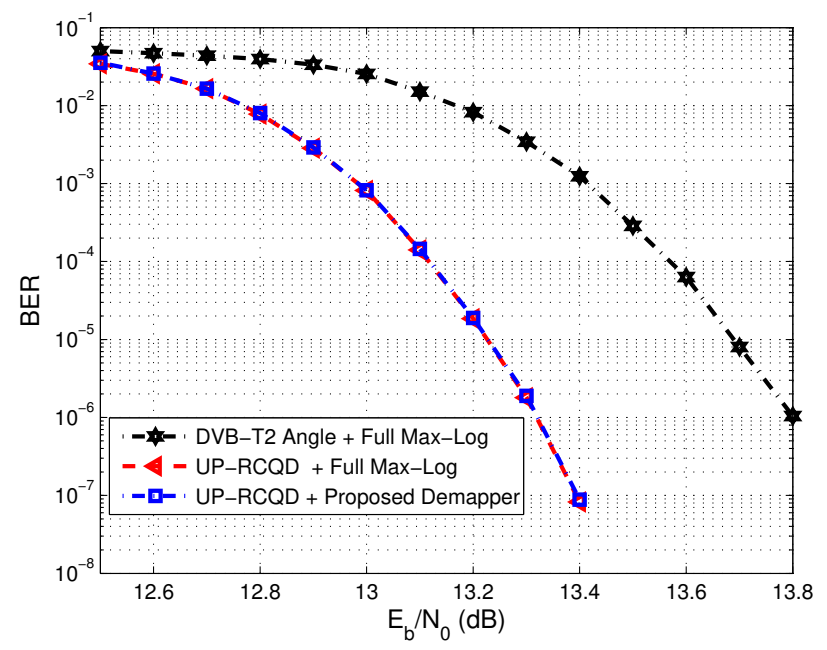

Figure 9. BER comparison between the UP-RCQD solution and the DVB-T2 angle for 16-QAM signals over Rayleigh fading channels with $15 \%$ of erasure events.

erasure events for QPSK, 16-QAM and 64-QAM respectively. In consistence with the theoretical results (Figs. 4, 7 and 10), Figs. 13, 14 and 15 show that the coding gain increases with the constellation size; for $\mathrm{CR}=5 / 6$, the coding gain with QPSK is roughly $0.15 \mathrm{~dB}$ at a $B E R=10^{-6}$, whereas it is about $1.4 \mathrm{~dB}$ for 64-QAM. Moreover, the coding gain increases with the CR; for example, for 16-QAM, the coding gain is approximately $0.2 \mathrm{~dB}$ for $\mathrm{CR}=1 / 2$, however it is about $0.8 \mathrm{~dB}$ for $\mathrm{CR}=5 / 6$. A larger gain is achieved for the erasure channel because the studied rotation angles maximize the minimum interval between two consecutive points projected over the real and the imaginary axis (see Property 1). Globally speaking, the structural properties of the examined rotation angles not only introduce a simplified demapping algorithm but also lead to improved robustness of the system to erasure events. Fig. 16 compares the performance obtained with the considered angle with that obtained with the angle $\alpha_{1}=\arctan ((1-\sqrt{5}) / 2)$ 


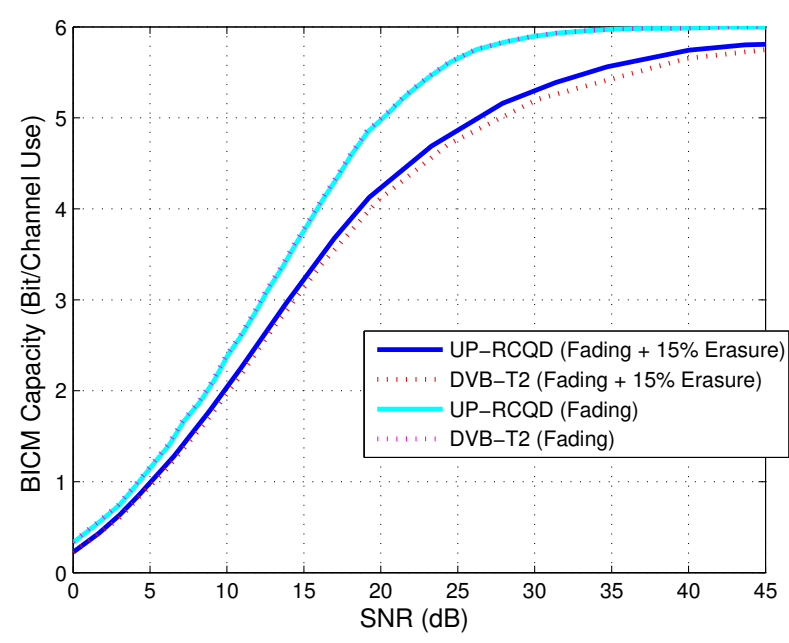

Figure 10. RCQD 64-QAM BICM capacity comparison between the UPRCQD solution and the DVB-T2 angle.

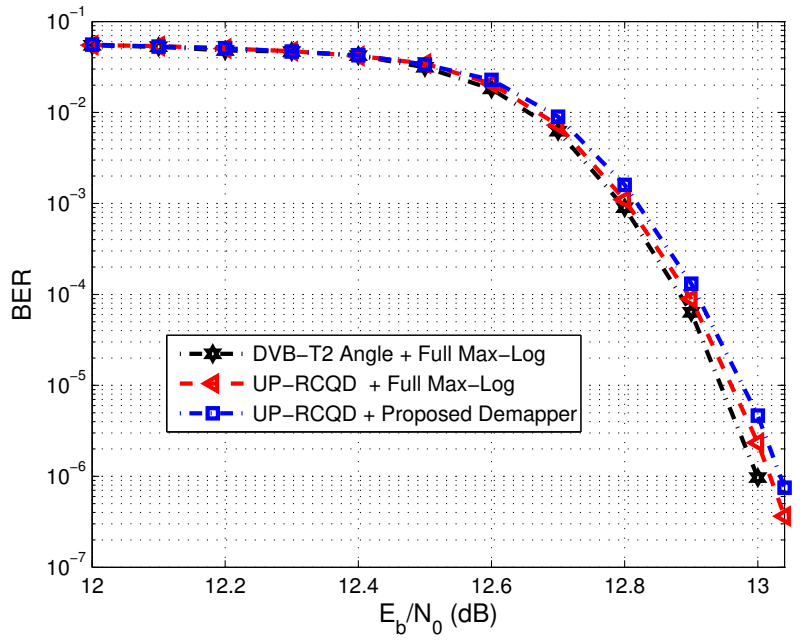

Figure 11. BER comparison between the UP-RCQD solution and the DVB-T2 angle for 64-QAM signals over Rayleigh fading channels.

[2] for 64-QAM signals over fading channel with and without erasure. $\alpha_{1}$ is obtained in [2] by maximizing the product distance between constellation points and it is often considered to be asymptotically optimal for rotated constellations. The performance obtained with the studied angle outperforms the performance obtained with $\alpha_{1}$ by $0.4 \mathrm{~dB}$ (resp. $5.5 \mathrm{~dB}$ ) over fading channels without erasure (resp. with $15 \%$ of erasure events). Notice that the angle originally proposed by [2] does not lead systematically to the best bit error rate in practical scenarios at finite SNR. Similar results can be obtained for other constellations.

Finally, it can be mentioned that for a lower erasure rate, the obtained performances in terms of error rates lies between that of the previously displayed cases: fading channel without any erasure and fading channel with $15 \%$ of erasure events. For instance, Fig. 17 compares the performance for the 64-QAM signal with the studied angle, $\alpha_{1}$ and the DVB-T2 rotation

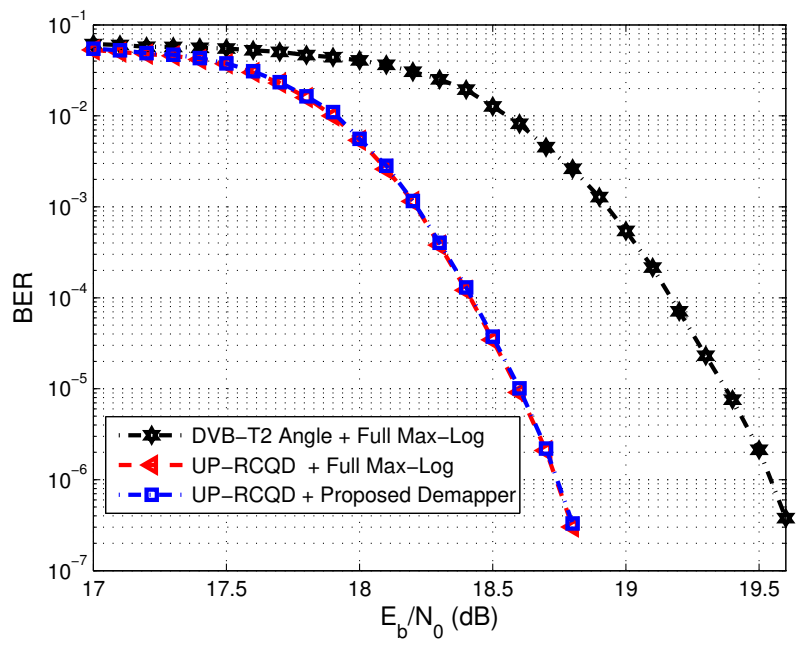

Figure 12. BER comparison between the UP-RCQD solution and the DVBT2 angle for 64-QAM signals over Rayleigh fading channel with $15 \%$ of erasure events.

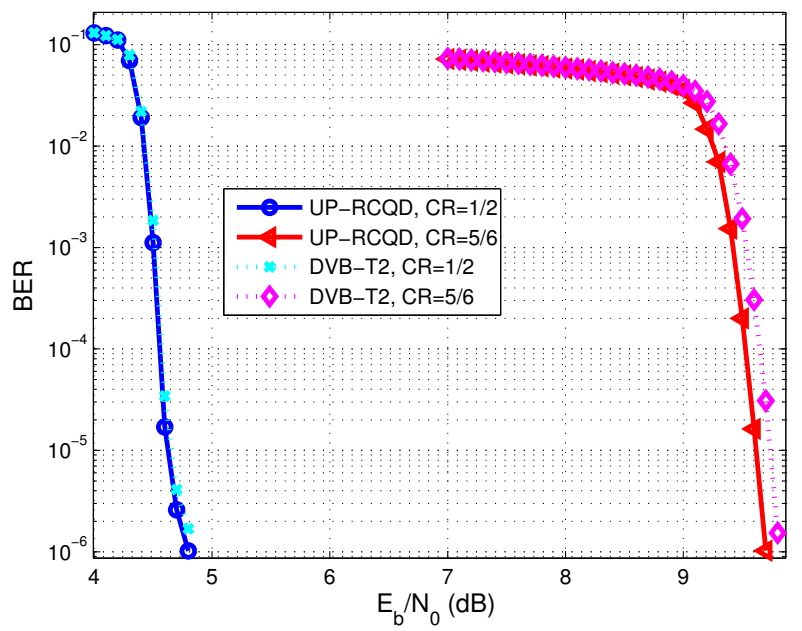

Figure 13. BER comparison between the UP-RCQD solution and the DVB$\mathrm{T} 2$ angle for QPSK signals with $\mathrm{CR}=1 / 2$ and $\mathrm{CR}=5 / 6$ over a Rayleigh fading channel with $15 \%$ of erasure events.

angle over fading channel with $5 \%$ of erasure events.

\section{B. The Proposed Sphere-Demapper}

This part compares in terms of BER and computation complexity, the proposed sphere-based demapping algorithm with other currently used methods including the Max-Log method [25], the MMSE method [12], the Sub-Region method [13] and the PD-DEM method [14].

Due to the fact that the rotation angle of the DVB-T2 RCQD signal is $\alpha=\arctan (1 / N)$ for the 256-QAM, the proposed demapper can also directly be applied to this case. Table I and Table II give the complexity comparison in terms of CP, RM, RS, RC and RI to demap one 256-QAM symbol, respectively without any erasure and with one component erased (see also Appendix A). 


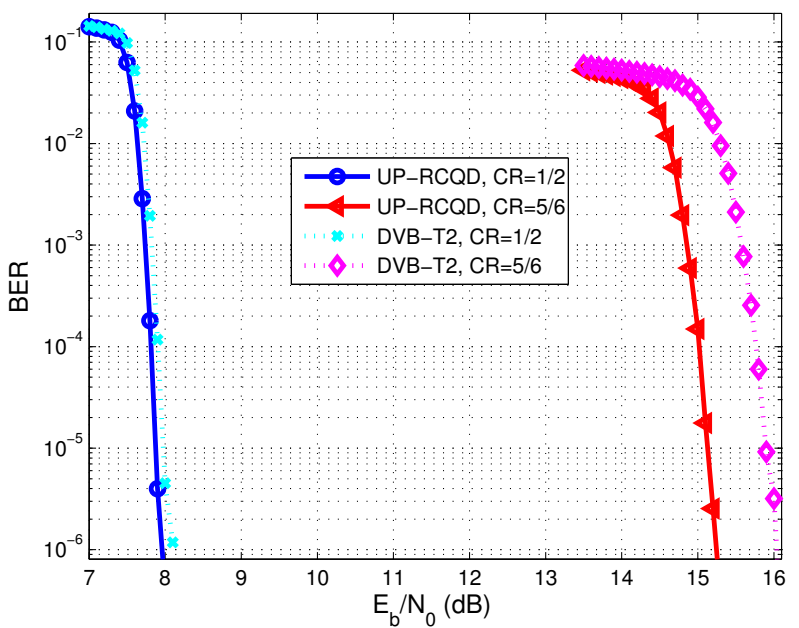

Figure 14. BER comparison between the UP-RCQD solution and the DVB-T2 angle for 16-QAM signals with $\mathrm{CR}=1 / 2$ and $\mathrm{CR}=5 / 6$ over a Rayleigh fading channel with $15 \%$ of erasure events.

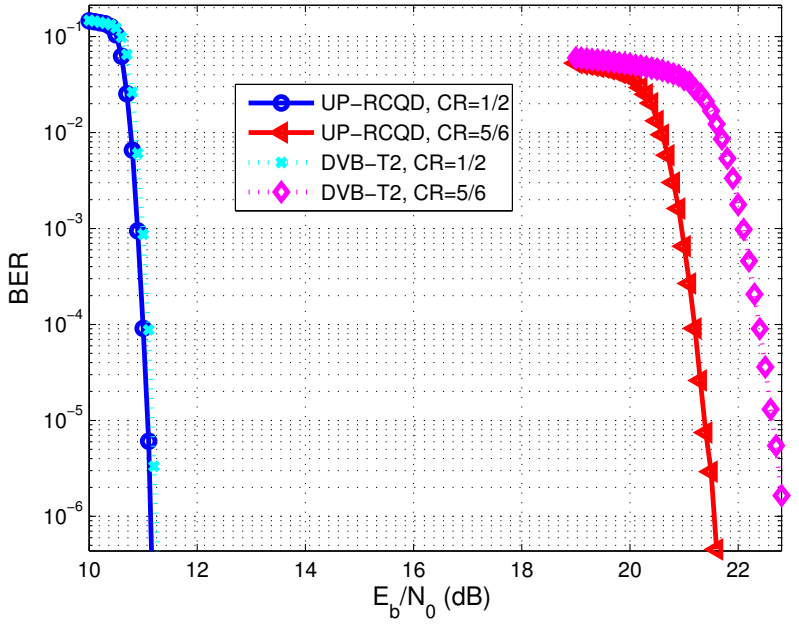

Figure 15. BER comparison between the UP-RCQD solution and the DVB-T2 angle for $64-\mathrm{QAM}$ signals with $\mathrm{CR}=1 / 2$ and $\mathrm{CR}=5 / 6$ over a Rayleigh fading channel with $15 \%$ of erasure events.

Table I

DEMAPPER COMPLEXITY WITHOUT ANY ERASURE EVENT FOR 256-QAM

\begin{tabular}{|c|c|c|c|c|c|}
\hline & CP & RM & RS & RC & RI \\
\hline \hline Max-Log & 256 & 1032 & 776 & 2032 & 0 \\
\hline Sub-Region & 81 & 332 & 251 & 632 & 0 \\
\hline MMSE & 16 & 64 & 48 & 112 & 6 \\
\hline PD-DEM & 80 & 390 & 279 & 241 & 0 \\
\hline Proposal & 32 & 138 & 106 & 275 & 2 \\
\hline
\end{tabular}

Table II

DEMAPPER COMPLEXITY WITH ERASURE EVENT FOR 256-QAM

\begin{tabular}{|c|c|c|c|c|c|}
\hline & CP & RM & RS & RC & RI \\
\hline \hline Max-Log & 256 & 520 & 264 & 2032 & 0 \\
\hline Sub-Region & 144 & 296 & 152 & 1136 & 0 \\
\hline MMSE & 16 & 54 & 43 & 112 & 5 \\
\hline PD-DEM & 80 & 230 & 119 & 241 & 0 \\
\hline Proposal & 16 & 43 & 28 & 60 & 1 \\
\hline
\end{tabular}

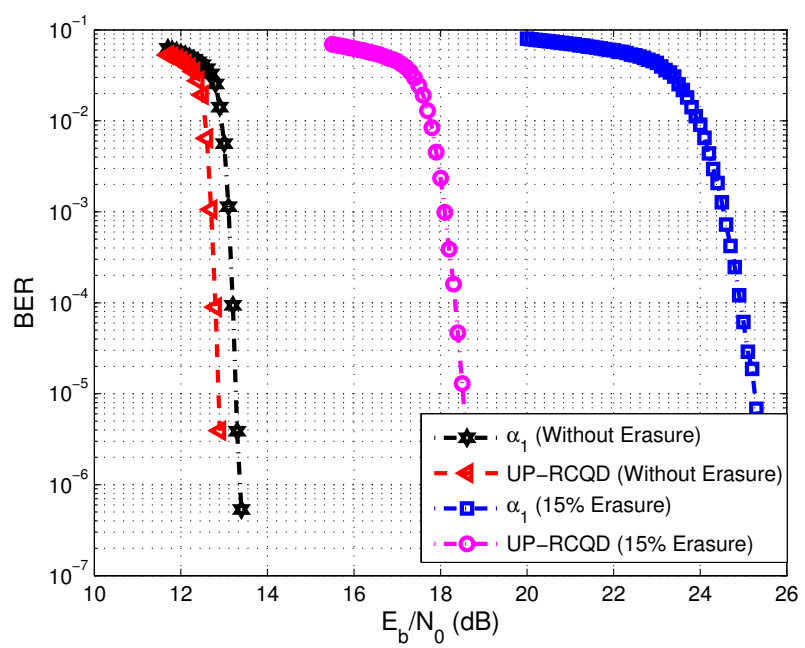

Figure 16. BER comparison between the UP-RCQD solution and the angle $\arctan ((1-\sqrt{5}) / 2)$ for 64-QAM signals.

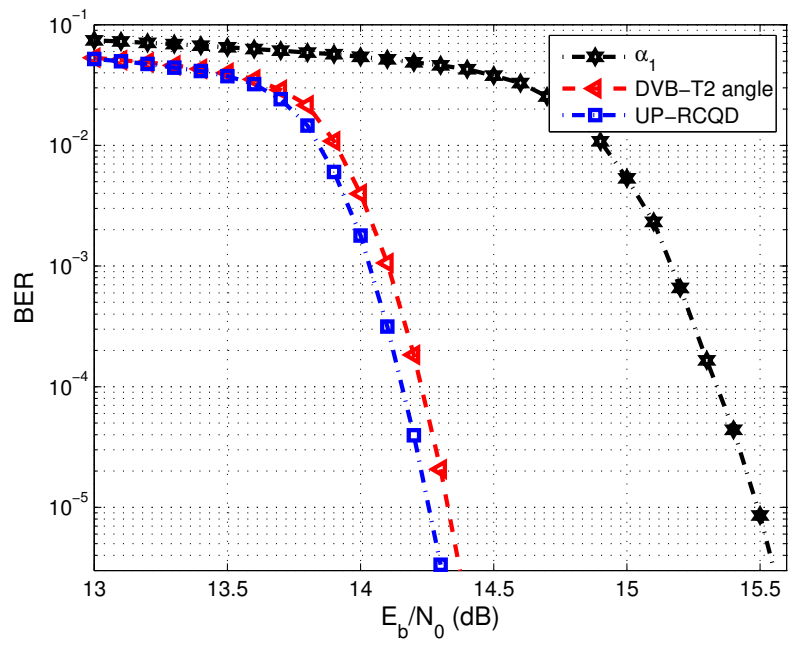

Figure 17. BER comparison between the UP-RCQD solution, $\arctan ((1-\sqrt{5}) / 2)$, and the DVB-T2 angle for 64-QAM signals over fading channel with $5 \%$ of erasure events.

Fig. 18 compares the BER performance of the various demapping algorithms for the fading channel without any erasure. The MMSE is the only method which has a lower computation complexity than our proposal; however, it is unable to achieve the optimum performance due to the fact that the decorrelation based algorithms are not optimum [24]. It can be observed that, of all studied algorithm, 2D joint detection methods can natively reach more easily a near optimum performance. Among them, the proposed method achieves almost the same performance as the full complexity Max-Log algorithm over a fading channel; however it reduces by $88 \%$ the number of CPs, $86 \%$ the numbers of RMs, $74 \%$ the number of RSs and $86 \%$ the number of RCs with respect to the full complexity Max-Log algorithm (see Table I). Furthermore, compared with the PD-DEM, the proposed demapping algorithm introduces a $60 \%$ reduction in number 


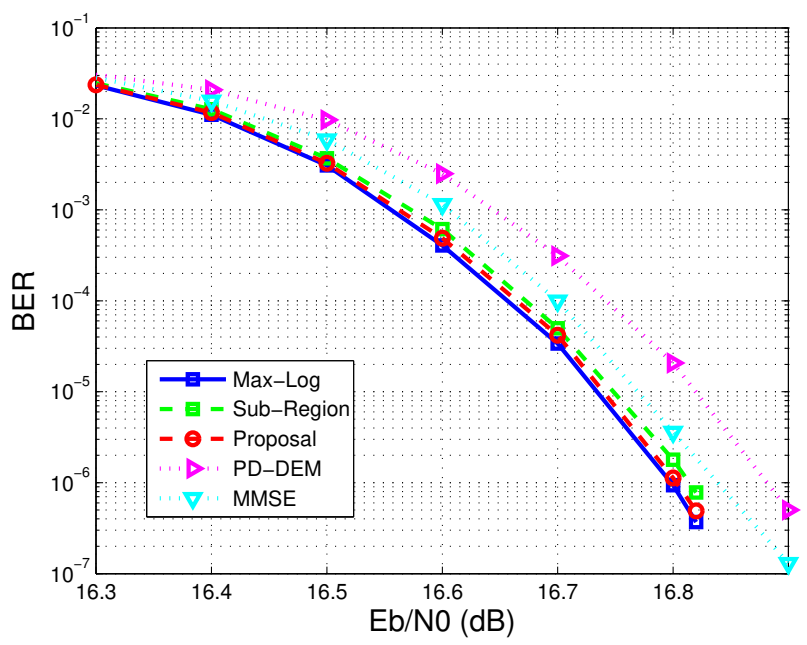

Figure 18. BER comparison of the considered methods for the DVB-T2 RCQD 256-QAM over a Rayleigh fading channel.

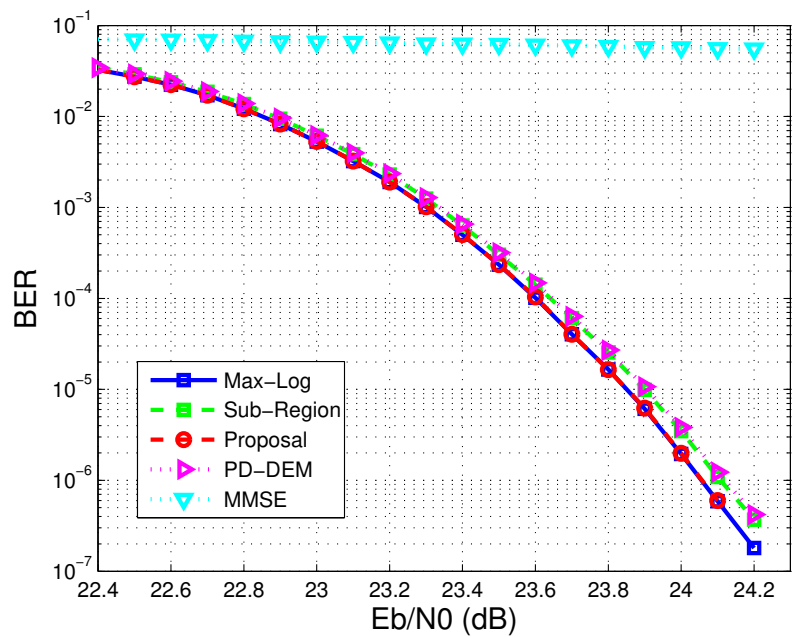

Figure 19. BER comparison of the considered methods for the DVB-T2 RCQD 256-QAM over a Rayleigh fading channel with $15 \%$ of erasure events.

of CPs and a 64\% reduction in number of RMs and achieves a better performance.

Fig. 19 compares the BER performance of the various demapping algorithms over a fading channel with erasure events. Among them, the MMSE method achieves the worst performance with a high error floor. Since the proposed algorithm is proved to systematically locate the ML solution and the corresponding complementary points, it is closely following the near optimum performance with even lower complexity. It reduces by $96 \%$ the number of CPs, $92 \%$ the number of RMs, $72 \%$ the number of RSs and $99 \%$ the number of RCs with respect to the full complexity Max-Log algorithm and only requires 1 additional RI (see Table II). Furthermore, the complexity of the proposed method is even lower than that of the MMSE method. In particular, Table II illustrates the power consumption reduction that can be obtained in case of erasure events.
Table III

DEMAPPER COMPLEXITY WITHOUT ANY ERASURE EVENT

\begin{tabular}{|c|c|c|c|}
\hline & CP & RM & RI \\
\hline \hline M1 & $N^{2}$ & $4 N^{2}+\log _{2} N^{2}$ & 0 \\
\hline M2 & $\left(\frac{N}{2}+1\right)^{2}$ & $4\left(\frac{N}{2}+1\right)^{2}+\log _{2} N^{2}$ & 0 \\
\hline M3 & $N$ & $2 N+\log _{2} N^{2}+24$ & 6 \\
\hline M4 & $N\left(\frac{\log _{2} N^{2}}{2}+1\right)$ & $N\left(2 \log _{2} N^{2}+8\right)+\log _{2} N^{2}-2$ & 0 \\
\hline
\end{tabular}

\begin{tabular}{|c|c|c|}
\hline & RS & RC \\
\hline \hline M1 & $3 N^{2}+\log _{2} N^{2}$ & $\left(N^{2}-2\right) \log _{2} N^{2}$ \\
\hline M2 & $3\left(\frac{N}{2}+1\right)^{2}+\log _{2} N^{2}$ & $\left(\left(\frac{N}{2}+1\right)^{2}-2\right) \log _{2} N^{2}$ \\
\hline M3 & $2 N+\log _{2} N^{2}+8$ & $(N-2) \log _{2} N^{2}$ \\
\hline M4 & $N\left(\frac{3}{2} \log _{2} N^{2}+5\right)+\log _{2} N^{2}-1$ & $3 N+\left(\frac{7}{4} N-\frac{7}{2}\right) \log _{2} N^{2}-3$ \\
\hline
\end{tabular}

\section{CONClusion}

This paper studies a series of rotation angles having interesting structural properties for different RCQD signals. Based on these properties, a low-complexity sphere-demapper is derived for both fading channels with and without erasure events. Some important advantages of the proposed demapper are as following; first, the sphere radius implies the number of constellation points involved to perform soft demapping. Second, it is always able to find the optimum point for the case with one (in-phase or quadrature) component erased. By applying the proposed solution to the DVB-T2 system and comparing with the original rotation angles, the UPRCQD approximately achieves the same performance as the original solution over fading channels without any erasure events for different constellations. In addition, better results are achieved over fading channels with erasure events for different constellations and this improves the system robustness. A critical advantage of the proposed solution is its very low complexity which simplifies both the transmitter and the receiver sides so that the proposed demapper is particularly suited for hardware implementation; this quality is totally independent from the OFDM modulation and from the channel coding [31]-[33] choice. Due to all these features, the UPRCQD M-QAM could be a modulation candidate for future terrestrial broadcasting systems, and even more, many wireless systems could take advantage of its signal space diversity, explored with a low complexity implementation.

\section{APPENDIX A}

\section{GENERAL COMPLEXITY CALCULATION OF OTHER METHODS}

The complexities of the other methods for the non-erasure channel and the one-component erasure channel, including the full-complexity Max-Log method labeled as M1, the SubRegion method labeled as M2 [13], the MMSE method labeled as M3 [12] and the PD-DEM method labeled as M4 [14], are displayed in Table III and Table IV.

\section{REFERENCES}

[1] L. Szczecinski, and A. Alvarado, Bit-Interleaved Coded Modulation: Fundamentals, Analysis and Design, John Wiley and sons, Chichester, UK, 2015. 
Table IV

DEMAPPER COMPLEXITY WITH ERASURE EVENTS

\begin{tabular}{|c|c|c|c|}
\hline & CP & RM & RI \\
\hline \hline M1 & $N^{2}$ & $2 N^{2}+\log _{2} N^{2}$ & 0 \\
\hline M2 & $\left(\frac{N}{2}+1\right) N$ & $2\left(\frac{N}{2}+1\right) N+\log _{2} N^{2}$ & 0 \\
\hline M3 & $N$ & $2 N+\log _{2} N^{2}+14$ & 5 \\
\hline M4 & $N\left(\frac{\log _{2} N^{2}}{2}+1\right)$ & $N\left(\log _{2} N^{2}+6\right)+\log _{2} N^{2}-2$ & 0 \\
\hline
\end{tabular}

\begin{tabular}{|c|c|c|}
\hline & RS & RC \\
\hline \hline M1 & $N^{2}+\log _{2} N^{2}$ & $\left(N^{2}-2\right) \log _{2} N^{2}$ \\
\hline M2 & $\left(\frac{N}{2}+1\right) N+\log _{2} N^{2}$ & $\left(\left(\frac{N}{2}+1\right) N-2\right) \log _{2} N^{2}$ \\
\hline M3 & $2 N+\log _{2} N^{2}+3$ & $(N-2) \log _{2} N^{2}$ \\
\hline M4 & $N\left(\frac{1}{2} \log _{2} N^{2}+3\right)+\log _{2} N^{2}-1$ & $3 N+\left(\frac{7}{4} N-\frac{7}{2}\right) \log _{2} N^{2}-3$ \\
\hline
\end{tabular}

[2] J. Boutros, and E. Viterbo, "Signal space diversity: a power- and bandwidth-efficient diversity technique for the Rayleigh fading channel," IEEE Trans. on Inform. Theory, 44(4):1453-1467, July 1998.

[3] X. Giraud, E. Boutillon, and J.C. Belfiore, "Algebraic tools to build modulation schemes for fading channels," IEEE Trans. on Inform. Theory, 43(3):938-952, May 1997.

[4] Q. Xie, J. Song, K. Peng, F. Yang, and Z. Wang, "Coded modulation with signal space diversity," IEEE Trans. on Wireless Comm., 10(2):660-669, Feb. 2011.

[5] J. Kim, W. Lee, J-K. Kim, and I. Lee, "On the symbol error rates for signal space diversity schemes over a Rician fading channel,"IEEE Trans. on Comm., 57(8):2204-2209, Aug. 2009.

[6] S. Jeon, J-Y. Choi, Z. Yim, and J-S. Seo, "Error floor analysis of signal space diversity schemes under erasure effect in single frequency network," IEEE Trans. on Broadcasting, 58(3):508-513, Sep. 2012.

[7] N. H. Tran, H.H. Nguyen, and T. Le-Ngoc, "Performance analysis and design criteria of BICM-ID with signal space diversity for keyhole Nakagami-m fading channels,"IEEE Trans. on Inform. Theory, 55(4):1592-1602, April 2009.

[8] N. H. Tran, H.H. Nguyen, and T. Le-Ngoc, "Application of signal space diversity over multiplicative fading channels," IEEE Signal Proc. Letters, 16(3):204-207, March 2009.

[9] Digital Video Broadcasting (DVB); frame structure, channel coding and modulation for a second generation digital terrestrial television broadcasting system (DVB-T2). European Tele. Standard Institute ETSI Yotk EN 302755 V1.1.1., Sep. 2009.

[10] C. Abdel Nour, and C. Douillard, "Rotated QAM constellation to improve BICM performance for DVB-T2," in Proc. IEEE 10th Inter. Sym. On Spread Spectrum Techniques and Application, ISSSTA 08, pp. 354-359, Aug. 2008.

[11] M. Li, C. Abdel Nour, C. Jego, J. Yang, and C. Douillard, "Efficient iterative receiver for Bit-Interleaved Coded Modulation according to the DVB-T2 standard," Inter. Conf. on Acoustics, Speech and Sig. Process. (ICASSP), Prague, 2011, pp. 3168-3171.

[12] K. Kim, K. Bae, and H. Yang, "One-dimensional soft-demapping using decorrelation with interference cancellation for rotated QAM constellation," in Proc. 2012 IEEE Con. Comm. and Net. Conference (CCNC), pp. 787-791, Jan. 2012.

[13] M. Li, C. Abdel Nour, C. Jego, and C. Douillard, "Design of rotated QAM mapper/demapper for the DVB-T2 standard," in Proc. IEEE work. on Sig. Proc. Sys. (SiPS), 2009, pp. 018-023, Oct. 2009.

[14] S. Tomasin, and M. Butussi, "Low complexity demapping of rotated and cyclic Q delayed constellation for DVB-T2," IEEE Wireless Comm. Letters, 1(2):81-84, April 2012.

[15] D. Perez-Calderon, V. Baena-Lecuyer, A.C. Oria, P. Lopez, and J.G Doblado, "Simplified rotated constellation demapper for second generation terrestrial digital video broadcasting," IEEE Trans. on Broadcasting, 59(1):160-167, March 2013

[16] J. Yang, M. Li, C.A. Nour, C. Douillard, B. Geller, "Max Log Demapper Architecture Design for DVB T2 Rotated QAM Constellations," Proceedings of IEEE SIPS, Hangzhou, October 2015.

[17] G. Taricco, and E. Viterbo, "Performance of component interleaved signal sets for fading channels," Elec. Letters, 32(13):1170-1172, June 1996.

[18] N.F. Kiyani, and J.H. Weber, "Performance analysis of a partially coherent system using constellation rotation and coordinate interleaving," in Proc. IEEE Global Telecom. Conference, GLOBECOM, 2008, pp. 1-5, Nov. 2008

[19] A. Chindapol, and J.A. Ritcey, "Bit-Interleaved Coded Modulation with signal space diversity in Rayleigh fading," In Proc. Conf. Record of the Thirty-Third Asilomar Conf. on Sig. Sys. and Comp., 1999, v. 2 pp. 1003-1007, Oct. 1999.

[20] N.F. Kiyani, U.H. Rizvi, J.H. Weber, and G.J.M. Janssen, "Rotation optimization for MPSK/MQAM signal constellation over Rayleigh fading channels," in Proc. IEEE Wireless Comm. And Net. Conf. WCNC 2007, pp. 997-981, March 2007.

[21] N.F. Kiyani, U.H. Rizvi, J.H. Weber, and G.J.M. Janssen, "Rotation optimization for MPSK/MQAM signal constellation over Rayleigh fading channels," in Proc. 10th IEEE Singapore Inter. Conf. on Comm. Sys., ICCS 2006, pp. 1-5, Oct. 2006.

[22] J. Yang, K. Wan, B. Geller, C. Abdel Nour, O. Rioul, and C. Douillard, "A low-complexity 2D signal space diversity solution for future broadcasting systems", in Proc. IEEE Inter. Conf. on Comm., ICC 2015, pp. 2762-2767, June 2015

[23] A. R. Jafri, A. Baghdadi, M. Waqas, and N. Ul-Islam, "High-Throughput and Area-Efficient Rotated and Cyclic Q Delayed Constellations Demapper for Future Wireless Standards," IEEE Access, 5 (1): 3077-3084, Jan. 2017.

[24] B. Hassibi, and H. Vikalo, "On the sphere-decoding algorithm I. Expected complexity," IEEE Trans. on Sig. Proc., 53(8):2806-2818, Aug. 2005.

[25] Implementation guidelines for a second generation digital terrestrial television broadcasting system (DVB-T2). European Telecom. Standard Institute ETSI TR 102831 V1.1.1, Oct. 2010.

[26] G. Caire, G. Taricco, and E. Biglieri, "Bit-Interleaved Coded Modulation," IEEE Trans. on Info. Theory, 44(3):927-946, May 1998.

[27] P. Xue, K. Bae, K. Kim, and H. Yang, " $\sqrt{M}$-best candidates based softdemapper for rotated M-QAM constellation," Consumer Communications and Networking Conference (CCNC), 2014 IEEE, pp. 623-928, Jan. 2014.

[28] D. Alfonso, D. Pérez-Calderón, J. García Doblado, P. López García, A. C. Oria Oria and V. Baena Lecuyer, "Análisis del Ángulo de Rotación en DVB-T2," Acta del XXV Simposium Nacional de la Unión Científica Internacional de Radio, Bilbao, URSI, Sept. 2010.

[29] Z. Wu and Z. Luo, "Further rotation modulation application," Document submission IEEE 802.11-10, July 2010.

[30] B. Geller, I. Diatta, J.P. Barbot, C. Vanstraceele, F. Rambeau, "Block Turbo Codes : From Architecture to Application," Proceedings of IEEE ISIT, Seattle, July 2006.

[31] E. Arikan, "Channel polarization: A method for constructing capacityachieving codes for symmetric binary-input memoryless channels," IEEE Transactions on Information Theory, pp. 3051-3073,Volume55(7), Jul. 2009.

[32] C. Vanstraceele, B. Geller, J.P. Barbot, J.M. Brossier, "A Low Complexity Block Turbo Decoder Architecture," IEEE Transactions on Communications, vol. 56, no 12, pp. 1985-1989, Dec. 2008.

[33] C Berrou, A Glavieux, "Near optimum error correcting coding and decoding: Turbo-codes," IEEE Transactions on communications vol. 44 no 10, pp. 1261-1271, 1996 